\title{
Aerodynamic interference on a finned slender body
}

\author{
Ross Chaplin ${ }^{\mathrm{a}}$ and David MacManus ${ }^{\mathrm{b}}$ \\ Cranfield University, Cranfield, Bedfordshire, MK43 OAL, UK
}

Friedrich Leopold $^{\mathrm{c}}$, Bastien Martinez ${ }^{\mathrm{d}}$ and Thibaut Gauthier ${ }^{\mathrm{e}}$

French-German Research Institute of Saint Louis, Saint-Louis, 68300, France

and

Trevor Birch ${ }^{\mathrm{f}}$

Defence Science and Technology Laboratory, Portsdown West, Hampshire, PO17 6AD, UK

Aerodynamic interference can occur between high-speed slender bodies when in close proximity. A complex flowfield develops where shock and expansion waves from a generator body impinge upon the adjacent receiver body and modify its aerodynamic characteristics in comparison to the isolated case. The aim of this research is to quantify and understand the multi-body interference effects which arise between a finned slender body and a second disturbance generator body. A parametric wind tunnel study was performed where the effects of receiver incidence and axial stagger were considered. Computational Fluid Dynamic simulations showed good agreement with the measurements and these were used in the interpretation of the experimental results. The overall interference loads for a given multi-body configuration are found to be a complex function of the pressure footprints from the compression and expansion waves emanating from the generator body as well as the flow pitch induced by the generator shockwave. These induced interference loads change sign as the shock impingement location moves aft over the receiver and in some cases cause the receiver body to become statically unstable. Overall, the observed interference effects can modify the subsequent body trajectories and may increase the likelihood of a collision.

$\mathrm{a} \quad=$ sonic velocity, $\mathrm{ms}^{-1}$

\section{Nomenclature}

\footnotetext{
${ }^{a} \mathrm{PhD}$ Research Student, Department of Aerospace Sciences, Member AIAA

b Senior Lecturer, Department of Aerospace Sciences, Member AIAA, Corresponding author, Tel: +44(0)1234754735, Email: d.g.macmanus@ cranfield.ac.uk

${ }^{\mathrm{c}}$ Aerodynamicist, French-German Research Institute of Saint Louis

${ }^{\mathrm{d}}$ Aerodynamicist, French-German Research Institute of Saint Louis

${ }^{\mathrm{e}}$ Aerodynamicist, French-German Research Institute of Saint Louis

${ }^{\mathrm{f}}$ Dstl Aerodynamics Fellow, Platform Systems Division, Senior Member AIAA
} 


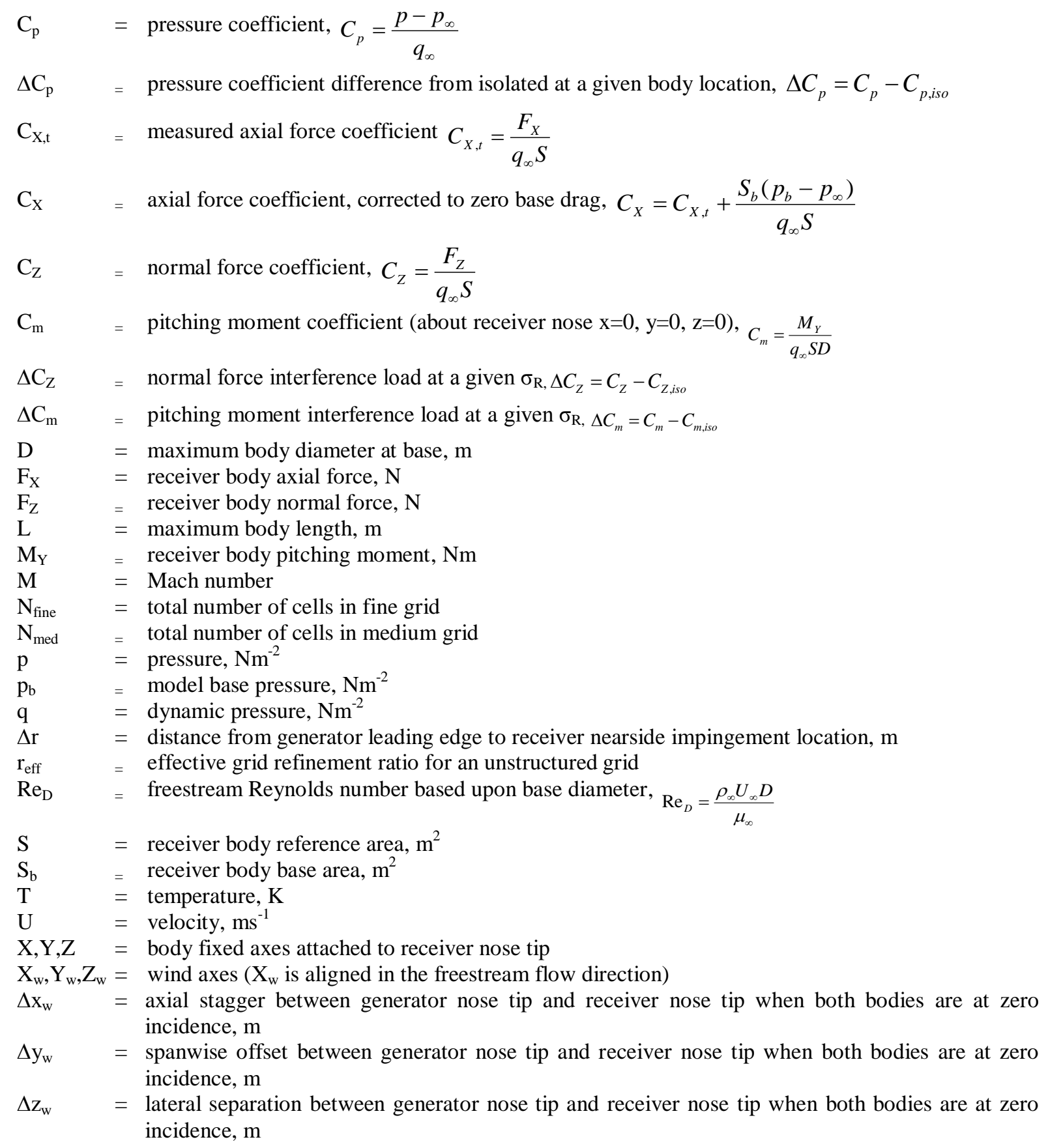

\section{Greek Symbols}

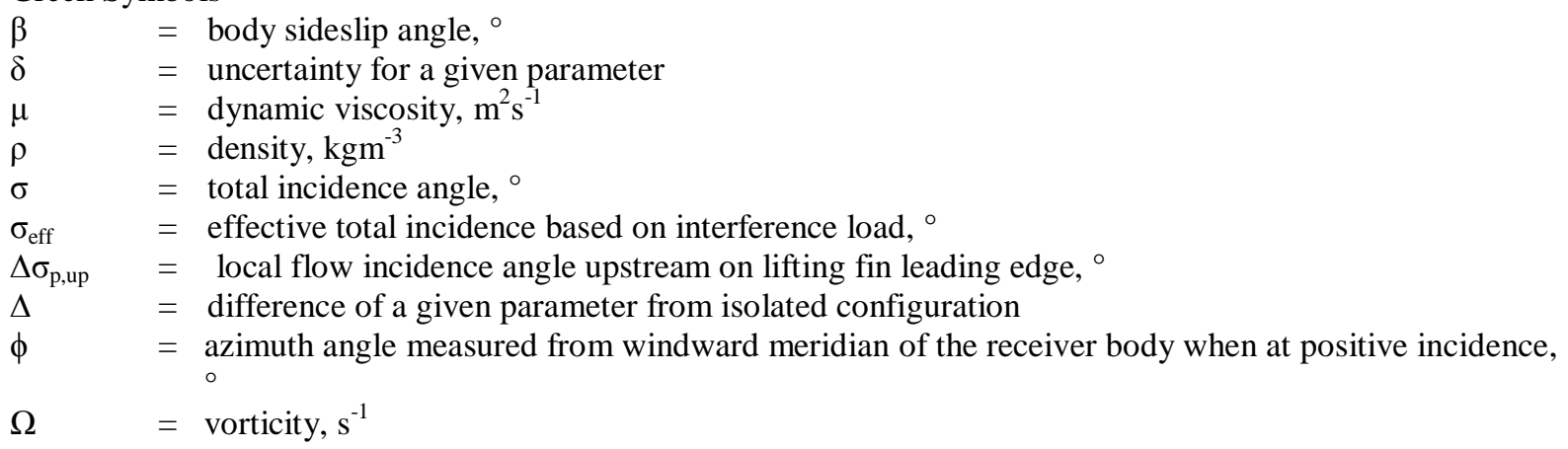

Subscripts

cg centre of gravity measured from receiver nose tip 


$\begin{array}{ll}\text { cp } & \text { centre of pressure measured from receiver nose tip } \\ \text { sm } & \text { static margin } \\ \text { near } & \text { receiver nearside conditions } \\ \text { iso } & \text { isolated conditions } \\ 0 & \text { stagnation conditions } \\ \infty & \text { freestream conditions } \\ \text { R } & \text { receiver body } \\ \text { G } & \text { generator body }\end{array}$

\section{Introduction}

ERODYNAMIC interference can occur when two bodies are placed in close proximity. For a supersonic flow the interference flowfield is dominated by shock and expansion waves which originate from an adjacent generator body and impinge upon the primary body of interest which is referred to as the receiver. This can affect the force and moment characteristics of the bodies and change their subsequent trajectories ${ }^{1-6}$. The induced changes in static pressure and flow angularity across the impinging disturbances modify both the local and overall aerodynamics of the receiver in comparison with the isolated body case. These interference effects can be particularly pronounced when the receiver body includes tail fins as the impinging disturbances can induce a local flowfield which causes large interference loads on these stability fins.

There is little information available in the open literature on the effects of mutual interference between similar slender bodies at high-speed. An early investigation by Gapcynski and Carlson ${ }^{3}$ reported normal force coefficient changes of up to $\Delta \mathrm{C}_{\mathrm{Z}}=0.1$ for two axially aligned un-finned bodies of revolution at $\mathrm{M}_{\infty}=2.01$. Within the wider context of high-speed interference research, another study, albeit at a relatively high Mach number of 6 compared with the current work, showed that a planar shock impinging on a cone-cylinder body at zero incidence induced changes in normal force and pitching moment coefficient in the range of $-0.22<\Delta \mathrm{C}_{\mathrm{Z}}<0.048$ and $-0.39<\Delta C_{m}<-0.20$ respectively ${ }^{1}$. The pitching moment values reported by Wilcox ${ }^{1}$ have been transformed to be consistent with the definition used throughout this paper. These changes were found to increase by up to an order of magnitude when the receiver body was placed at an incidence of $\sigma=15^{\circ}$. Low-order computational studies have been performed to model the supersonic interference aerodynamics between a submunition and a dispenser body ${ }^{7,8}$ and two computation approaches were used to estimate the forces and moments on the submunition. The effect of horizontal and lateral spacing, submunition incidence and the dispenser cavity was considered for a range of configurations across the range of $\mathrm{M}_{\infty}$ from 1.2 to 3.0. In general, despite some notable differences for particular configurations, the measured trends were captured by the computational method. Although for these configurations the submunition is much smaller than the parent dispenser body, the results highlighted important sensitivities of the normal force and pitching moment to the relative position and submunition angle of attack. In particular it was noted that the normal force and pitching moment changed sign 
depending on the position of the submunition. A more recent study, which investigated two axially aligned unfinned slender bodies at zero incidence, found that the sign of the resulting pitching moment was strongly dependent upon the initial lateral separation between the bodies ${ }^{9}$. It has been mooted by several authors that the mutual interference flowfield, and resulting induced pitching moment, could potentially have a notable effect on the trajectory of both bodies $3,4,10-12$.

In addition to the research which discusses the overall interference loads, it is important to understand the detailed underlying flow physics. Of particular interest are the shock-body interactions which have been studied previously for a number of configurations ${ }^{9,10,13,15} \cdot$ Brosh $^{16}$ and Hung ${ }^{2}$ investigated a wedge-generated shock impinging on a cylinder at $\mathrm{M}_{\infty}=3$ and showed that the shock footprint, in terms of local pressure rise, decreased as the shock diffracted around the body. This difference between the strength of the nearside and farside regions of augmented pressure significantly affected the local normal force distribution over the body. Finally, the nearside pressure rise also resulted in a local boundary-layer separation and a double-reflected shock structure around the primary shock-induced separation bubble. Both studies found that due to the induced circumferential pressure gradient, a strong crossflow occurred which resulted in a local separation on the farside of the receiver body. A similar effect was also noted by Morkovin et $\mathrm{al}^{17}$.

In addition to the mainly experimental studies discussed above, there have been some computational studies of mutual interference for slender bodies at high speeds. Hung ${ }^{18,19}$ conducted one of the few computational fluid dynamics (CFD) validation exercises using a thin-layer approximation of the 3D Reynolds Averaged Navier Stokes (RANS) equations to simulate the experimental set-up tested by Brosh ${ }^{16}$. This configuration featured a planar shock impinging onto a cylinder which was at zero incidence. Hung focussed on assessing the ability of the CFD solver to calculate the complex shockwave boundary-layer interactions around the cylinder. It was reported that the calculation of surface pressure distributions on the cylinder were in good agreement with the measurements. However, some of the viscous flow structures were not calculated well by the CFD. In particular, the flow topology on both the nearside and farside of the cylinder, which included induced flow separations, were not accurately calculated. More recently, Volkov ${ }^{9}$ investigated the effect of lateral separation between two axially aligned slender bodies and found reasonably good agreement between the measured and calculated loads. Finally, both Malmuth ${ }^{10}$ and Fedorov ${ }^{15}$ have reported theoretical approaches to the calculation of overall loads on two slender bodies in close proximity.

Although a few examples exist which investigate the interference effects between simple un-finned bodies of revolution or cylinders; and the related field of store separation, typically at transonic speeds, is well established, 
there is a dearth of literature focussing on aerodynamic interference at supersonic speeds for more realistic slender body configurations which are likely to include stability fins. The current research ${ }^{6,22,24}$ is focussed on addressing this gap. The aim of this paper is to quantify and to understand the multi-body interference effects which arise between a finned slender body and an adjacent generator body. This aim is addressed through a comprehensive wind tunnel study where the primary measurements included the receiver body forces and moments, surface pressures as well as shadowgraph visualizations of the flowfield. The experiments investigated the effect of the receiver body incidence as well as the axial stagger between the bodies. Given the complexity of the interference flowfield, supporting CFD calculations were used to help develop an understanding of the underlying causes of the force and moment measurements. This approach aims to use a mutually enhancing combination of experimental and computational results to develop a deeper understanding of the underlying flow physics.

\section{Experimental method}

\section{A. Wind tunnel and model arrangements}

The wind tunnel tests were performed using two bodies placed in close proximity. The body of interest is designated as the receiver, on which quantitative measurements were taken. The receiver comprises a 3.5D tangent ogive forebody and a cylindrical afterbody. It has an overall length of $\mathrm{L} / \mathrm{D}=7.358$, where $\mathrm{D}$ is the base diameter of $\mathrm{D}=20 \mathrm{~mm}$ (Fig. 1). The receiver body also includes a set of cruciform tail fins which were designed such that the body is marginally stable over an incidence range of $-15 \leq \sigma \leq 15^{\circ}$. These tail fins are rectangular in planform with a hexagonal thickness profile $(0.2 \mathrm{c}, 0.6 \mathrm{c}, 0.2 \mathrm{c})$, a chord of 1 caliber $(\mathrm{c} / \mathrm{D}=1)$, a semi-span equal to $\mathrm{b} / \mathrm{D}=0.65$ and a maximum thickness to chord ratio (t/c) of 0.1 (Fig. 1).

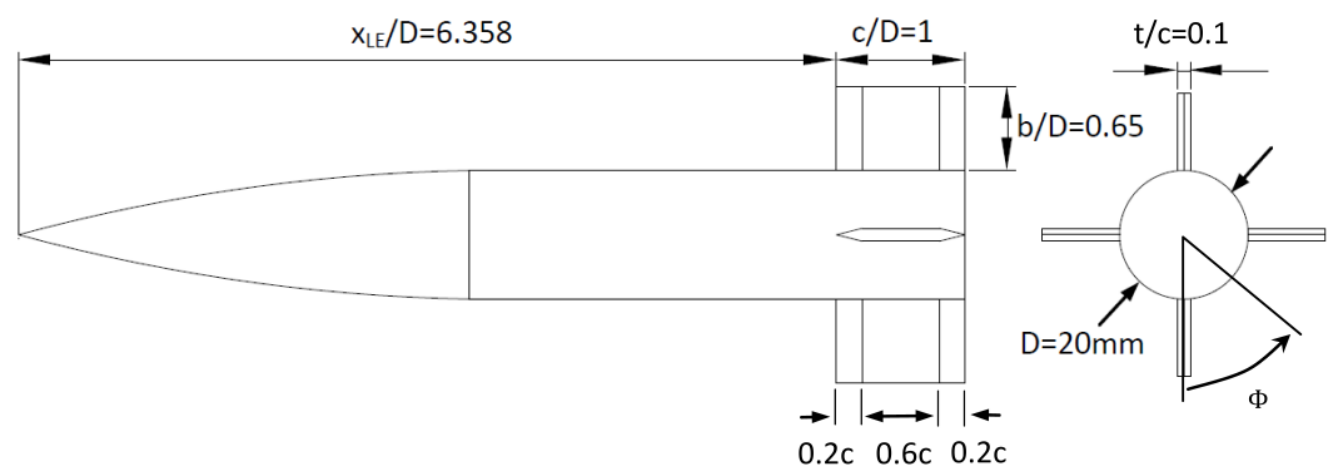

Fig. 1 Schematic of the receiver body and tail fins 
The other body is denoted as the generator and this is used to create the disturbance flowfield. Two axisymmetric generator bodies were used. The first is a sharp nosed generator which is the same as the receiver body but with no fins (Fig. 2a). The second generator is a blunt-nosed version of the sharp generator which has the same overall length as the sharp generator $(\mathrm{L} / \mathrm{D}=7.358, \mathrm{D}=20 \mathrm{~mm})$ but includes a hemi-spherical forebody (Fig. 2b) .

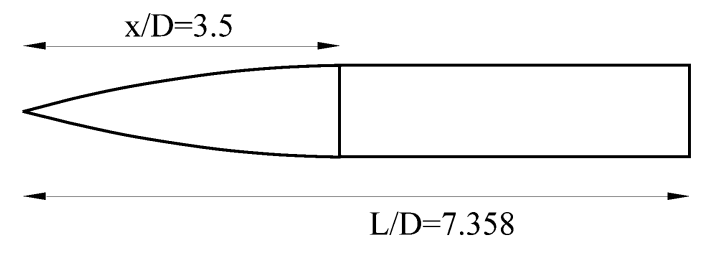

(a) Sharp generator

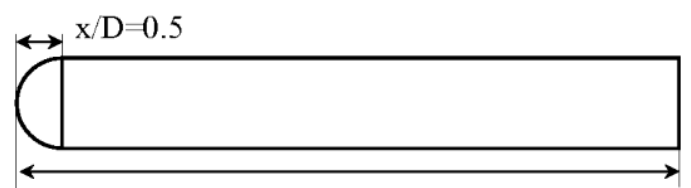

$\mathrm{L} / \mathrm{D}=7.358$

(b) Blunt generator

Fig. 2 Schematic of the generator bodies and definition of $\phi$

The experiments were performed in the $0.2 \mathrm{~m} \times 0.2 \mathrm{~m}$, S20 supersonic wind tunnel at the French-German Research Institute of Saint-Louis (ISL). This is a blowdown tunnel with a typical run time of 50s. The stagnation conditions in the settling chamber were a total pressure of $\mathrm{p}_{0}=0.675 \mathrm{MPa}$ and a total temperature of $\mathrm{T}_{0}=293 \mathrm{~K}$. The stagnation chamber supplied a rectangular nozzle which produced nominal freestream flow conditions in the working section with a Mach number of $\mathrm{M}_{\infty}=2.43$ and a Reynolds number, based on body base diameter, of $\operatorname{Re}_{\mathrm{D}}=1.4 \times 10^{6}$. No boundary-layer transition devices were used for the interference tests presented in this study using the finned receiver body. However, additional measurements on an un-finned isolated receiver body were performed as part of the research programme using a wire transition strip which was attached $2 \mathrm{~mm}$ from the leading edge. These tests demonstrated a negligible effect on the normal force and pitching moment coefficients with only a small increase in axial force of $\Delta \mathrm{C}_{\mathrm{X}, \mathrm{t}}=0.01$ in comparison to the natural transition tests ${ }^{6}$. It was therefore considered that the boundary-layer was naturally turbulent during these experiments.

Multi-body testing was performed using a dual model support system (Fig. 3). A traverse mechanism allowed both the upper generator body and the lower receiver body to be translated relative to one another in the streamwise direction. The incidence of the receiver body was controlled using the lower support sting and 
quadrant, which rotated about a center of rotation point $1.65 \mathrm{D}$ upstream of the base on the body centerline. Each body was connected to their respective supports using a $D_{s}=16 \mathrm{~mm}$ diameter sting (Fig. 3).

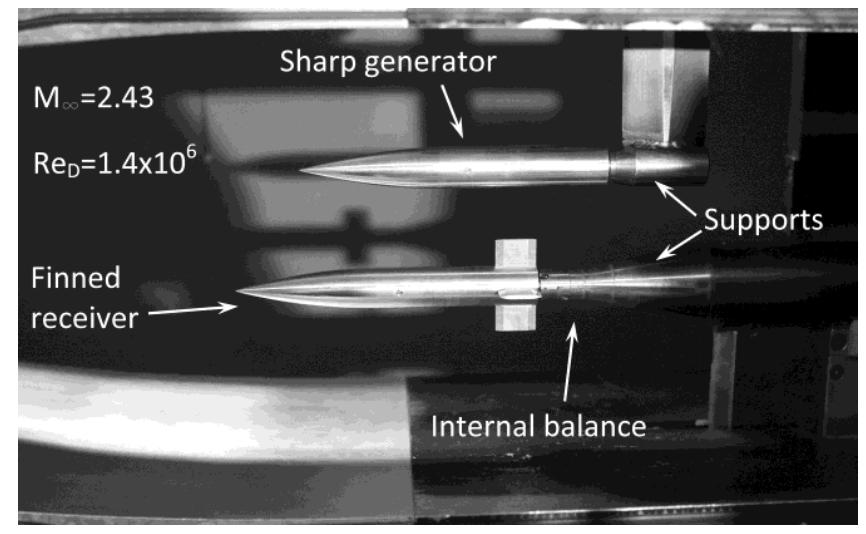

Fig. 3 Wind tunnel arrangement of finned receiver and sharp generator

Each multi-body configuration is defined by the relative lateral separation $\left(\Delta \mathrm{z}_{\mathrm{w}}\right)$, spanwise offset $\left(\Delta \mathrm{y}_{\mathrm{w}}\right)$ and axial stagger $\left(\Delta \mathrm{x}_{\mathrm{w}}\right)$ between the receiver and generator nose tips when both bodies are at zero incidence (Fig. 4$)$. The spanwise offset between the bodies and the body sideslip angles were both zero in all cases $\left(\Delta y=0, \beta=0^{\circ}\right)$ and the lateral separation $\left(\Delta \mathrm{z}_{\mathrm{w}} / \mathrm{D}=2.94\right)$ and generator incidence $\left(\sigma_{\mathrm{G}}=0^{\circ}\right)$ also remained fixed throughout. The receiver body was always orientated in the zero roll position $\left(\lambda=0^{\circ}\right)$ which is the plus ' + ' fin orientation relative to the tunnel axis.

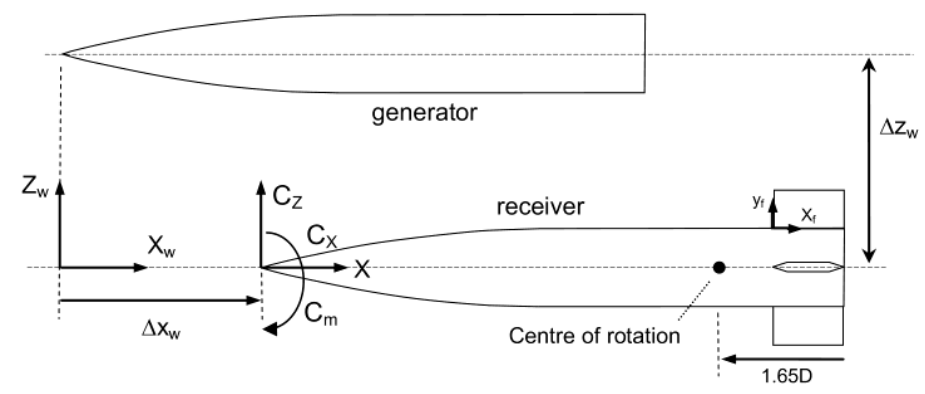

Fig. 4 Definitions of positive axial stagger $\left(\Delta \mathbf{x}_{\mathrm{w}}\right)$ and positive lateral separation $\left(\Delta \mathbf{z}_{\mathrm{w}}\right)$ between the receiver and generator nose tips.

\section{B. Experimental method}

For the multi-body configurations, the receiver body incidence was typically varied between $-15 \leq \sigma_{R} \leq 15^{\circ}$. A 5s settling period was employed at the start of each tunnel run. At each incidence, the receiver body paused and the forces and moments were measured using an ABLE MKXIV 6-component, internal balance. The force and moment balance output was filtered using a $5 \mathrm{~Hz}$ low-pass filter and the data was acquired at a sample rate of $100 \mathrm{kHz}$ with a typical sample duration of $2 \mathrm{~s}$. A shadowgraph image was also taken at each incidence setting. A 
light source was focused using a $0.38 \mathrm{~m}$ diameter lens and the illuminated flowfield was projected onto a transparent screen on the opposite tunnel window where a still image was taken with a PCO Sensicam qe camera. This same experiment procedure was used for different axial stagger configurations, different generator bodies as well as the isolated receiver configurations. The overall test matrix comprised configurations with an axial stagger of $\Delta x_{w} / D=-1.65,0,1.67,2.68$, a receiver incidence range of $-15 \leq \sigma_{R} \leq 15^{\circ}$ as well as the use of the sharp and blunt generator bodies. Finally, Pressure Sensitive Paint (PSP) measurements on the receiver were taken for a subset of configurations at $\Delta \mathrm{x}_{\mathrm{w}} / \mathrm{D}=1.67$ and a full description of the PSP set-up and measurement procedure is presented in Chaplin et $\mathrm{al}^{23}$.

\section{Measurement uncertainty}

The experimental uncertainties were calculated using the approach of Taylor ${ }^{25}$ and are mostly expressed as a fractional uncertainty of the measured value (Table 1). All measurement uncertainties were estimated from systematic and random sources of error which included the instrument calibration, instrument accuracy given by the manufacturer and the data acquisition resolution. Based on the isolated receiver measurements, a flow angularity correction of $\sigma_{\mathrm{cor}}=-0.05^{\circ}$ was applied to the measured data which ensured zero normal force at zero incidence for the isolated configuration.

Table 1 Summary of the measurement uncertainties

\begin{tabular}{|c|c|c|c|c|c|c|}
\hline \multicolumn{2}{|c|}{ Tunnel arrangement } & \multicolumn{2}{|c|}{ Freestream conditions } & \multicolumn{2}{|c|}{ Forces and moments } & \multirow{2}{*}{$\begin{array}{l}\text { PSP } \\
\mathrm{p} \pm 10 \%\end{array}$} \\
\hline$\Delta \mathrm{x}_{\mathrm{w}} / \mathrm{D}$ & $\pm 0.7-5.7 \%$ & $\mathrm{M}_{\infty}$ & $2.43 \pm 1.2 \%$ & $\mathrm{C}_{\mathrm{Z}}, \mathrm{C}_{\mathrm{m}}$ & $\pm 0.6 \%$ & \\
\hline$\Delta \mathrm{z}_{\mathrm{w}} / \mathrm{D}$ & $\pm 0.1 \%$ & $\operatorname{Re}_{\mathrm{D}}$ & $1.4 \times 10^{6} \pm 0.7 \%$ & $\mathrm{C}_{\mathrm{X}}$ & $\pm 2.5 \%$ & \\
\hline \multirow[t]{2}{*}{$\sigma_{\mathrm{R}}$} & $\pm 0.1^{\circ}$ & & & $\Delta \mathrm{C}_{\mathrm{Z},}, \Delta \mathrm{C}_{\mathrm{m}}$ & $\pm 0.9 \%$ & \\
\hline & & & & $\Delta \mathrm{C}_{\mathrm{X}}$ & $\pm 3.5 \%$ & \\
\hline
\end{tabular}

\section{Computational method}

\section{A. Solver description}

All computational calculations were conducted using the Cobalt flow solver ${ }^{26}$. This is an unstructured, implicit code based on a finite volume formulation and further details are reported by Strang ${ }^{27}$. Steady-state viscous calculations were performed which solved the Reynolds averaged Navier-Stokes (RANS) equations and modeled turbulence using Menter's 2-equation SST turbulence model ${ }^{28}$. The solutions were obtained using $2^{\text {nd }}$ order spatial accuracy and the wind tunnel freestream conditions were specified as initial conditions.

\section{B. Computational grids}


All the 3D computational grids were created using the grid generation software Gridgen ${ }^{29}$ and were based on a hybrid meshing approach. The computational domain modeled one-half of the flowfield since the sideslip angles for both bodies were zero. This assumption was supported by the measurements of side force and yawing moment for the receiver body which were found to be nominally zero. The computational domain extended from a short distance upstream of the leading edge of the foremost body to approximately 3D downstream of the base of the aftmost body (Fig. 5a). The base and supporting sting were also included for each body where appropriate. The surrounding flowfield domain boundary was arranged so that the bow shock of both bodies exited through the downstream outlet face.

The hybrid grids contained both structured and unstructured blocks and used hexahedral, tetrahedral and pyramid cell types. The body surfaces, and a region close to each body, were gridded using structured cells in order to achieve an appropriate level of boundary-layer resolution and appropriate control of the grid refinement regions, particularly between the fins (Fig. 5b). A first cell spacing in the radial direction adjacent to each solid wall was chosen to ensure an average $\mathrm{y}^{+}$value over the body of less than or equal to one ${ }^{30}$. The typical first cell wall normal distance was $3.4 \times 10^{-6} \mathrm{~m}$. A progression ratio of approximately 1.1 was used to cluster the grid points towards the body surface and typically 25-30 cells spanned the boundary-layer maximum thickness ${ }^{30}$. External to the near-surface structured zone were two unstructured zones. The unstructured zone adjacent to the structured zone contained cells which were similar in size to the structured cells. The farfield unstructured zone was coarser since it was far from the bodies. Special care was taken to ensure there was smooth cell size progression across all block boundaries.

\section{Boundary Conditions}

All body and sting surfaces were defined as adiabatic, no-slip, solid walls (Fig. 5a). A symmetry condition was applied on the $\mathrm{x}_{\mathrm{w}}-\mathrm{Z}_{\mathrm{w}}$ plane since the sideslip angles of the bodies were zero. Both the inlet and farfield boundary conditions were prescribed as fixed supersonic inflows at the wind tunnel freestream operating conditions. The outlet boundary values were calculated using a modified Riemann invariants condition ${ }^{26}$. 


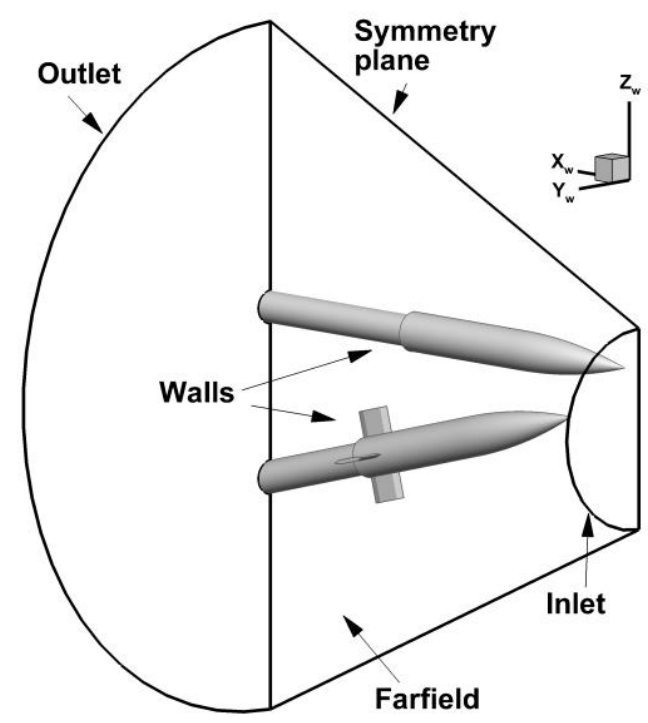

(a)computational domain

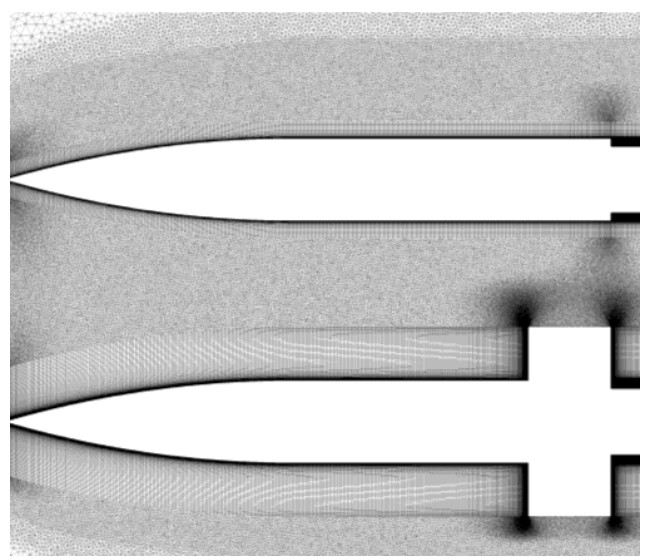

(b) sample of grid

Fig. 5 Computational model(a) domain and boundary conditions (b) sample of grid on the $x_{w}-z_{w}$ symmetry plane

\section{Computational uncertainty}

\section{Iterative convergence}

Iterative convergence was assessed by examination of the solution residuals and the forces and moments on the receiver body over the solution history. For all computational solutions, satisfactory iterative convergence of the solution residuals was observed and the forces and moments converged to within $0.5 \%$ of the reported values.

\section{Grid convergence}

A selection of configurations which are representative of the dataset as a whole were further investigated to assess the solution sensitivity to spatial resolution and to provide an estimate of the discretization error using the approach of Roache ${ }^{31}$. A grid convergence study was completed for each of the selected configurations over 
three grid levels. For the hybrid grids used in this investigation it was necessary to use an effective grid refinement ratio $^{31}$. This was based on the relative total grid sizes and was approximately $\mathrm{r}_{\text {eff }} \approx 1.5$ for this investigation (Eqn. (1) shows $r_{\text {eff }}$ between the fine and medium grid levels).

$$
r_{\text {eff }}=\left(\frac{N_{\text {fine }}}{N_{\text {med }}}\right)^{1 / 3}
$$

The integrated force and moment coefficients $\left(\mathrm{C}_{\mathrm{X}}, \mathrm{C}_{\mathrm{Z}}, \mathrm{C}_{\mathrm{m}}\right)$ were used as the comparison parameters in the grid convergence study. Typical values of Grid Convergence Index (GCI), as defined by Roache ${ }^{31}$, between the fine and medium grids were less than $0.5 \%$ in this paper. Consequently, the fine grid solutions presented in this paper were considered to be grid independent. Typical grid sizes were in the order of 18 to 22 million cells.

\section{Results}

The flow physics involved in the multi-body configurations is often highly complex and the elemental flow features tend to be bespoke to each configuration. This makes the extraction of simple and general trends difficult. To develop the necessary understanding of the aerodynamic effects, this section initially focuses on the receiver in isolation and then goes on to discuss the simpler multi-body configurations where the receiver incidence is zero. Further analysis is then devoted to configurations where the receiver is at incidence and where the aerodynamics becomes more complex. Finally, the effect of the axial stagger between the bodies and receiver incidence are also assessed.

\section{A. Aerodynamics of the receiver body in isolation}

In advance of assessing the complexities of the multi-body configurations, the aerodynamics of the receiver body in isolation is first considered with regard to the force and moment characteristics as a function of incidence. Key aspects of the flowfield which are pertinent to the subsequent analysis of the multi-body configurations are also discussed.

\section{Receiver force and moment characteristics as a function of incidence}

As expected, for the receiver body in isolation, both normal force $\left(\mathrm{C}_{\mathrm{Z}}\right)$ and pitching moment $\left(\mathrm{C}_{\mathrm{m}}\right)$ show a predominately linear variation with incidence $\left(\sigma_{\mathrm{R}}\right)$ (Fig. 6a). The pitching moment reference point is located at the receiver nose tip. At an incidence of $\sigma_{R}=15^{\circ}$, the CFD results show that the fins account for approximately a third (35\%) of the total normal force and for half (49\%) of the total pitching moment (Table 2). Overall, there is good agreement between the measured and calculated values of $\mathrm{C}_{\mathrm{Z}}$ and $\mathrm{C}_{\mathrm{m}}$. For a mid-range nominal incidence of $\sigma_{R}=6^{\circ}$ there is a difference of $1.9 \%$ between the calculated and measured $C_{z}$ and $-0.5 \%$ for $C_{m}$. However, in 
the high negative incidence regime, there is a more noticeable discrepancy between the measured and calculated loads. The measurements of $C_{Z}$ are a maximum of $7 \%$ larger than the calculations at the extreme value of $\sigma_{R}=-$ $15^{\circ}$. There are small variations in the measured corrected axial force coefficient across the incidence range (Fig. 6a). However, the CFD calculations underestimate the magnitude of the measured $\mathrm{C}_{\mathrm{x}}$ by an average of $11 \%$ across the incidence range and these calculated values lie outside the stated experimental uncertainty. Moreover, the calculated values show that the fins account for approximately half of the total axial force loads for most incidence angles (Table 2). The measured values of lateral forces and moments are all nominally zero and this supports the decision to perform the numerical simulations using an assumption of centerline symmetry.

Table 2 CFD calculated composition of force and moment coefficients for the isolated receiver body and fins at $\sigma_{\mathrm{R}}=0,8,15^{\circ}$.

\begin{tabular}{|c|ccc|ccc|ccc|}
\cline { 2 - 10 } \multicolumn{1}{c|}{} & \multicolumn{3}{c|}{$\boldsymbol{\sigma}_{\mathrm{R}}=\mathbf{0}^{\circ}$} & \multicolumn{3}{c|}{$\boldsymbol{\sigma}_{\mathrm{R}}=\mathbf{8}^{\circ}$} & \multicolumn{3}{c|}{$\boldsymbol{\sigma}_{\mathrm{R}}=\mathbf{1 5}^{\circ}$} \\
\cline { 2 - 10 } & $\mathrm{C}_{\mathrm{x}}$ & $\mathrm{C}_{\mathrm{z}}$ & $\mathrm{C}_{\mathrm{m}}$ & $\mathrm{C}_{\mathrm{x}}$ & $\mathrm{C}_{\mathrm{z}}$ & $\mathrm{C}_{\mathrm{m}}$ & $\mathrm{C}_{\mathrm{x}}$ & $\mathrm{C}_{\mathrm{z}}$ & $\mathrm{C}_{\mathrm{m}}$ \\
\hline body & 0.18 & 0.00 & 0.00 & 0.18 & 0.59 & -2.00 & 0.18 & 1.59 & -6.16 \\
fin & 0.15 & 0.00 & 0.00 & 0.15 & 0.55 & -3.68 & 0.17 & 0.86 & -5.81 \\
total & 0.33 & 0.00 & 0.00 & 0.33 & 1.14 & -5.68 & 0.35 & 2.45 & -11.97 \\
\hline
\end{tabular}
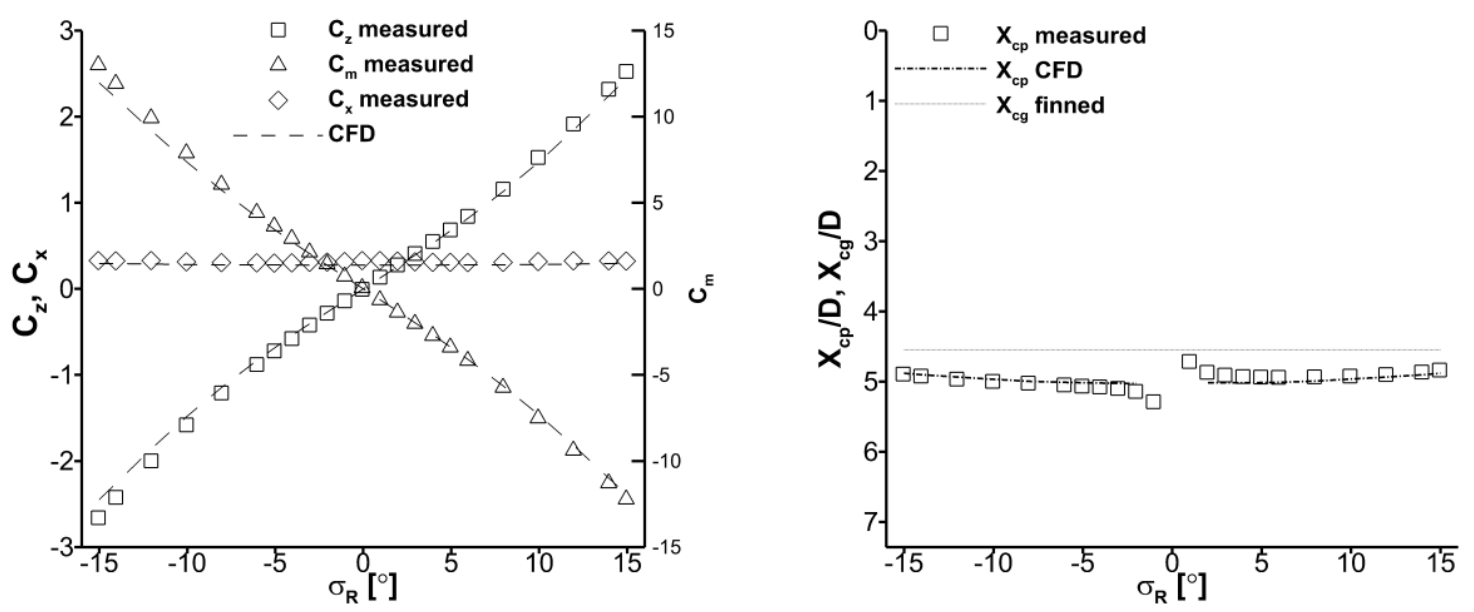

Fig. 6 (a) Calculated and measured normal force, axial force and pitching moment coefficients as a function of incidence for the finned receiver body in isolation (b) Longitudinal center of pressure location as a function of incidence for the receiver body in isolation.

Across the incidence range investigated $\left(-15 \leq \sigma_{R} \leq 15\right)$, the longitudinal center of pressure location $\left(\mathrm{X}_{\mathrm{cp}}\right)$ of the receiver body remains approximately fixed at $\mathrm{X}_{\mathrm{cp}} / \mathrm{D} \approx 5$ (Fig $6 \mathrm{~b}$ ). The centre of pressure and centre of gravity are measured from the receiver nose tip and $X_{c p}$ is calculated from $X_{c p}=-C_{m} / C_{N}{ }^{32}$. Importantly, $X_{c p}$ is aft of the center of gravity location $\left(\mathrm{X}_{\mathrm{cg}} / \mathrm{D}=4.55\right)$ and results in a positive static margin, meaning that the body is statically 
stable over the incidence range studied. A typical magnitude of the marginal static margin is $\mathrm{X}_{\mathrm{sm}} / \mathrm{D}=0.3$ for a receiver incidence of $\sigma_{R}= \pm 15^{\circ}$. Moreover, good agreement is observed between the measured and calculated values of $\mathrm{X}_{\mathrm{cp}}$ with a maximum difference in the order of 0.1 calibers but frequently much less (Fig 6b).

\section{Flowfield features of the receiver body at $\sigma_{R}=0,8,15^{\circ}$}

Overall, there is a consistency between the measured and calculated forces and moments which forms a useful foundation on which to investigate in more detail the calculated flowfield for receiver incidence angles of $\sigma_{R}=0,8,15^{\circ}$. The aim of this is to establish a basis for subsequent analyses with which to understand how the interference flowfield affects the detailed aerodynamics of the receiver body. Although the reported fin loads in this paper include all four fins, the subsequent detailed analysis focuses on the lifting fins. Considering the symmetry about the $\mathrm{X}-\mathrm{Z}$ axis, in the rest of this paper these are usually referred to as a lifting fin rather than fins.

The leading edge shock structure on the lifting fin is important as it influences the surface pressures on both the fin itself as well as on the body in the inter-fin region. When the receiver incidence is zero, the strength of the upper and lower portions of the shock emanating from the leading edge of the lifting fin are equal. Both of these shocks intersect with the equivalent shocks emanating from the fins located on the $\mathrm{x}-\mathrm{z}$ symmetry plane at $\phi=0,180^{\circ}$. This is highlighted in Fig. 7a which shows the surface static pressure distributions on the receiver in side view as well as the receiver unwrapped surface in the $\mathrm{x} / \mathrm{L}-\phi$ plane. The receiver azimuth angle $(\phi)$ is measured from the windward meridian of the receiver body when at positive incidence, which in the current configuration is on the receiver body farside at the $\mathrm{X}-\mathrm{Z}$ symmetry plane (Fig. 4). These intersecting shocks lead to a high pressure zone in the inter-fin region which is bound by both shocks and is observed in the axial distributions of pressure on the body taken at $\phi=45,135^{\circ}$ (Fig. 8 (a), (b)). Since the effect of the fins on the body are equal in the upper and lower inter-fin regions there is no change in resultant local normal force in this region of the body.

As the receiver incidence increases, the lower portion of the lifting fin leading edge shock strengthens and the upper portion weakens (Fig. 7 (b),(c)). The augmented shock strength leads to regions of elevated pressure in the lower inter-fin region (Fig. 7(a),(c)). The opposite is observed in the upper inter-fin region where the local shock footprint decreases in magnitude as incidence increases (Fig. 8(b),(c)). However, the shock angle at which the shock propagates increases for the lower portion of the leading edge shock as the incidence increases and therefore the net effect on the body depends on the combined aspects of both the changes in shock strength and affected area. 


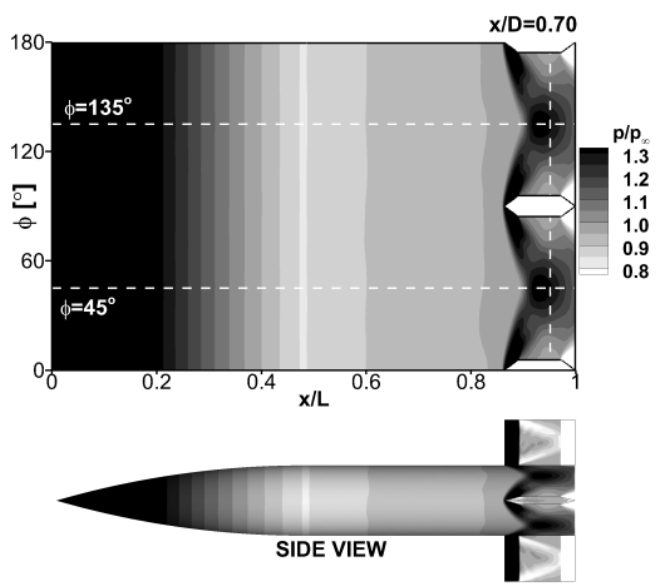

(a) $\sigma_{R}=0^{\circ}$

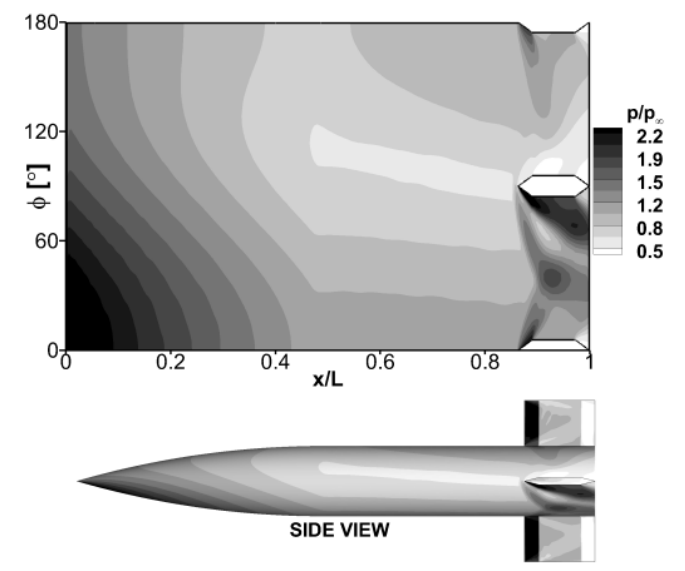

(b) $\sigma_{R}=8^{\circ}$

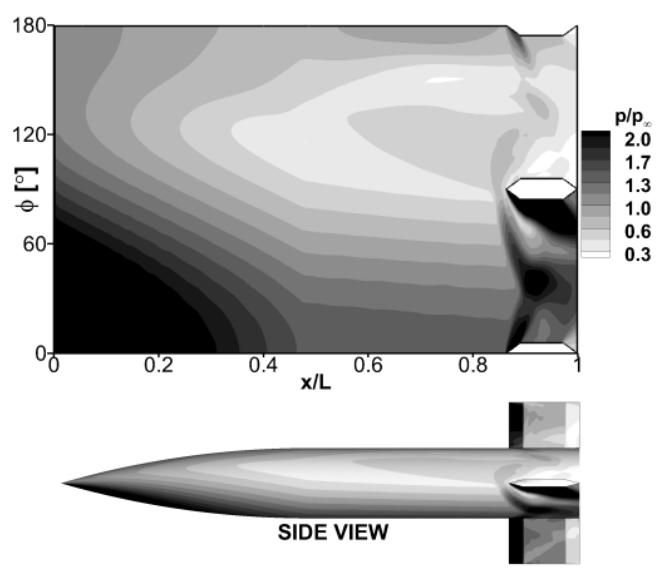

(b) $\sigma_{\mathrm{R}}=15^{\circ}$

Fig. 7 Calculated surface pressure contours for different isolated receiver incidence configurations (a) $\sigma_{R}=0^{\circ}(b) \sigma_{R}=8^{\circ}$ (c) $\sigma_{R}=15^{\circ}$. Note the different contour levels which are chosen for individual clarity. 


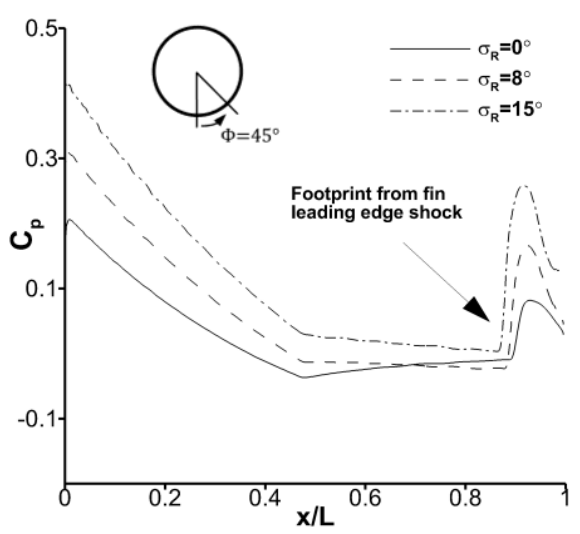

(a) axial distribution of pressure coefficient on farside quarter $\left(\phi=45^{\circ}\right)$

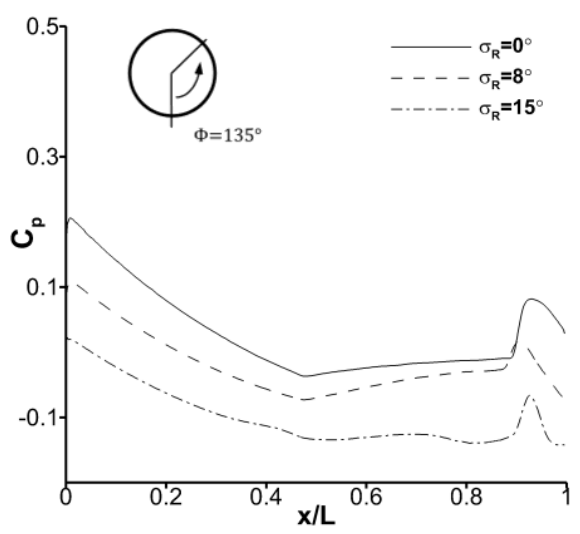

(b) axial pressure distribution on nearside quarter $\left(\phi=135^{\circ}\right)$

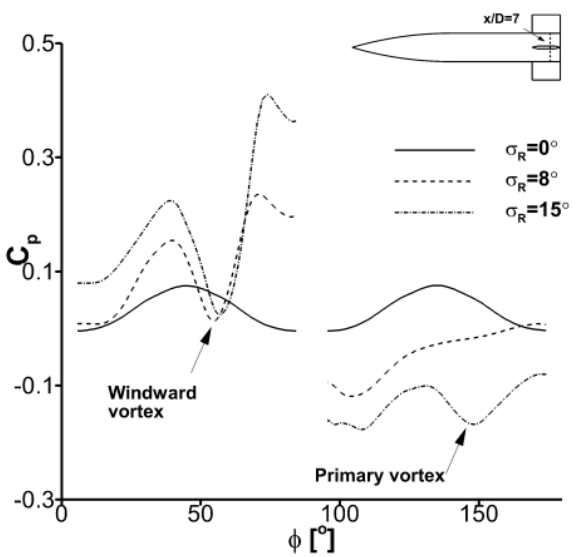

(c) circumferential pressure distribution at $\mathrm{x} / \mathrm{D}=7$

Fig. 8 Calculated isolated receiver surface pressure distributions along different planes (a-c) and for different incidence angles

As the receiver incidence increases from zero to $\sigma_{R}=15^{\circ}$, the local flow from the windward to leeward side of the body leads to a crossflow separation which is common for slender body configurations at moderate 
incidence. At $\sigma_{\mathrm{R}}=15^{\circ}$ the skin friction lines in Fig. 9(a) show a well-established and definitive primary separation line which feeds the primary vortex. There is also a smaller secondary body vortex which is located beneath the primary vortex which rotates in an opposite sense to the primary vortex. These two vortices cause a reduction in leeside pressure (Fig. 8) and give rise to the so-called 'vortex-lift' component of the overall normal force.

Of particular note is a third vortical flow feature adjacent to the lifting fin on the windward side of the body. A shock-induced separation vortex develops along the windward side of the body in the lower inter-fin region for the receiver incidence settings considered of $\sigma_{R}=8$ and $15^{\circ}$. Since the body is symmetrical, the vortical flow features are also observed at negative incidence. This separation is the result of a glancing shock interaction between the leading edge shock from the lower surface of the lifting fin and the local boundary-layer (Fig. 9 (a)). This flow feature is not observed at $\sigma_{R}=0^{\circ}$. As the incidence increases, so does the strength of the leading edge shock from the lower surface of the lifting fin and can be seen to a lesser extent at $\sigma_{R}=8^{\circ}$. When the receiver incidence is $\sigma_{R}=15^{\circ}$, this leads to a well-defined separation line (Fig. 9(b)) from which the local flow separates and rolls up into a small vortex close to the surface. The windward vortex is not as large as the primary vortex but it does have a noticeable effect on the inter-fin pressure distribution (Fig. 8 (c)) and acts to reduce the contribution of the lower inter-fin region to positive normal force.

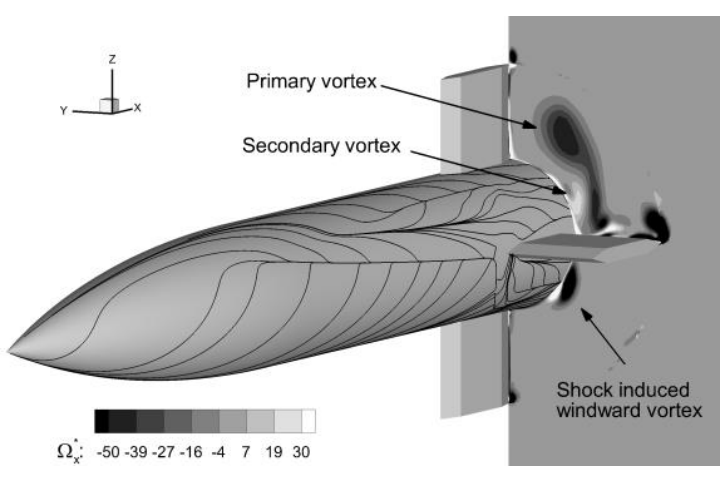

(a) oblique view of full body

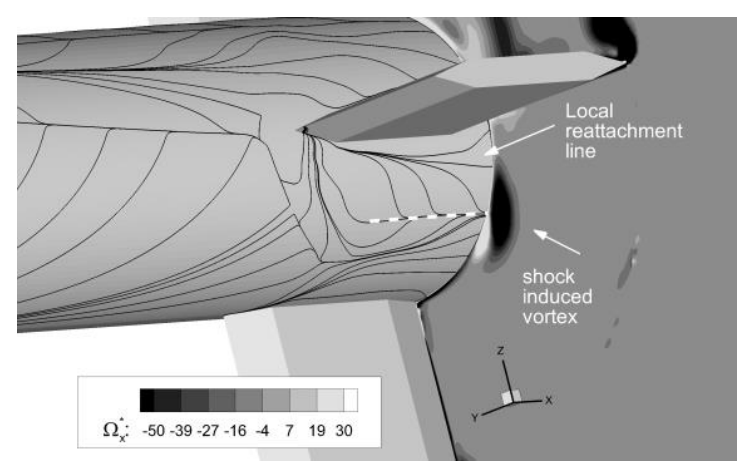

(b) close-up of the windward vortex highlighting local flow separation line (dashed) 
Fig. 9 Calculated surface skin friction vector lines and contours of $\Omega_{x}{ }^{*}$ on a crossflow slice at $x / L=1$ for the isolated receiver at $\sigma_{\mathrm{R}}=15^{\circ}$ (a) full body (b) close view of windward inter-fin region.

\section{B. Effect of axial stagger}

In multi-body configurations, the disturbance flowfield includes a primary shockwave which emanates from the generator forebody and impacts on the receiver body. It also includes the associated expansion wave which arises downstream of the primary shock. The axial impingement location of the primary shockwave is known to have a marked effect on the magnitude and sign of the interference loads acting on an un-finned slender body ${ }^{6,22,24}$. The fins on the receiver body in the current investigation were expected to intensify this effect. In this section, the interference aerodynamics for a selection of key cases from the measurement database are investigated which includes configurations with the sharp generator and the finned receiver at five different incidence angles $\left(\sigma_{R}=-15,-8,0,8,15^{\circ}\right)$ placed in four axial stagger arrangements $\left(\Delta x_{w} / D=-1.65,0,1.67,2.68\right)$. The calculated flowfields are then used to further understand the relevant flow physics for a subset of configurations at $\sigma_{R}=0^{\circ}$.

\section{Effect of axial stagger on the receiver force and moment characteristics}

The receiver interference loads are sensitive to the axial stagger between the bodies and both the normal force and pitching moment coefficients show a change in sign over the axial stagger range considered (Fig 10). Non-monotonic distributions of both normal force and pitching moment interference are observed as the sharp generator moves aft from upstream of the receiver $\left(\Delta \mathrm{x}_{\mathrm{w}} / \mathrm{D}=2.68\right)$ to downstream of the receiver $\left(\Delta \mathrm{x}_{\mathrm{w}} / \mathrm{D}=-1.65\right)$. The generator induces the largest magnitude of interference loads when the bodies are axially aligned $\left(\Delta \mathrm{x}_{\mathrm{w}} / \mathrm{D}=0\right)$. Broadly for all settings of receiver incidence $\left(\sigma_{R}=-15,-8,0,8,15^{\circ}\right)$, the observed interference loads $\left(\Delta \mathrm{C}_{\mathrm{z}}, \Delta \mathrm{C}_{\mathrm{m}}\right)$ show the same characteristics with an initial increase in interference magnitude as the generator moves from upstream to downstream with the local magnitude maximum at $\Delta \mathrm{x}_{\mathrm{w}} / \mathrm{D}=0$. 

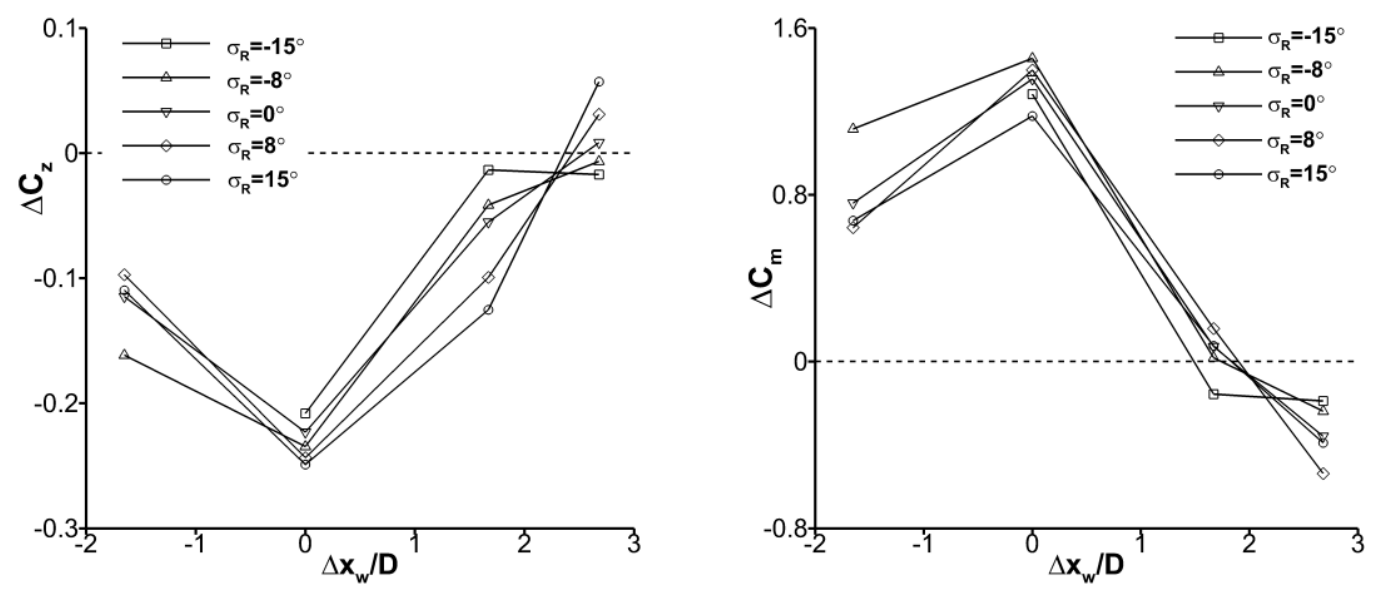

Fig. 10 Effect of axial stagger for $\Delta z_{w} / D=2.94, \sigma_{G}=0^{\circ}$ for $\sigma_{R}=-15,-8,0,8,15^{\circ}$ on (a) measured receiver normal force interference load (b) measured receiver pitching moment interference load.

2. Aerodynamics of the datum configuration $\left(\Delta x_{w} / D=0, \Delta z_{w} / D=2.94\right)$

The underlying aerodynamics of the multi-body configurations studied in this paper are highly complex. Before analyzing the force and moment trends above, this section introduces a datum configuration to identify and to explain the important flow physics and to describe some key metrics and concepts which will help interpret the results presented in the remainder of this paper.

The datum configuration comprises the receiver and sharp generator which are axially aligned $\left(\Delta \mathrm{x}_{\mathrm{w}} / \mathrm{D}=0\right)$, with a lateral separation of $\Delta \mathrm{z}_{\mathrm{w}} / \mathrm{D}=2.94$ and both bodies at zero incidence. An important characteristic of this configuration is that the impinging bow shock passes close to the lifting fins as it diffracts around the receiver body (Fig. 11). A first step in the analysis is to evaluate the relative contributions to the overall interference loads of the receiver body (ogival forebody and cylindrical centrebody) compared to the fins, which is done using the computed flowfield. The CFD calculations are essential to understand the detailed force and moment characteristics of the multi-body configurations since the individual fin loads were not measured in the experiments due to the size of the model. In this datum configuration, the fins account for $53 \%$ of the total calculated normal force interference and $58 \%$ of the pitching moment interference (Table 3 ). This underlines the potential effect that the fins can have on the magnitude of the interference loads for a multi-body case. 


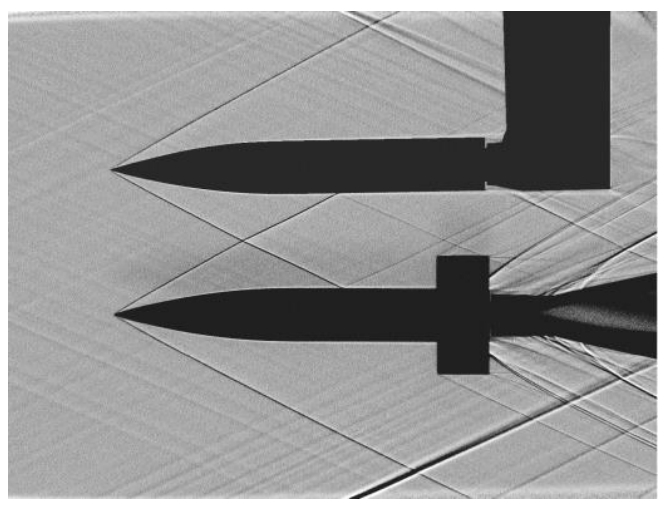

Fig. 11 Measured shadowgraph visualization for the datum finned configuration: sharp generator, $\Delta \mathbf{x}_{\mathrm{w}} / \mathrm{D}=\mathbf{0} \Delta \mathrm{z}_{\mathrm{w}} / \mathrm{D}=2.94, \sigma_{\mathrm{R}}=0^{\circ} \sigma_{\mathrm{G}}=0^{\circ}$

Table 3 Calculated receiver interference loads for the datum configuration $\Delta x_{w} / D=0 \Delta z_{w} / D=2.94, \sigma_{R}=0^{\circ}$ $\sigma_{\mathrm{G}}=\mathbf{0}^{\circ}$

\begin{tabular}{ccccccc}
\hline$\Delta \mathbf{x}_{\mathrm{w}} / \mathrm{D}$ & $\boldsymbol{\Delta} \mathrm{C}_{\mathrm{z}, \text { bod }}$ & $\boldsymbol{\Delta} \mathrm{C}_{\mathrm{z}, \text { fin }}$ & $\boldsymbol{\Delta} \mathrm{C}_{\mathrm{z}}$ & $\Delta \mathrm{C}_{\mathrm{m}, \text { bod }}$ & $\boldsymbol{\Delta} \mathrm{C}_{\mathrm{m}, \text { fin }}$ & $\Delta \mathrm{C}_{\mathrm{m}}$ \\
\hline $\mathbf{0}$ & -0.108 & -0.122 & -0.23 & 0.598 & 0.814 & 1.412
\end{tabular}

The overall interference loads are a complex function of different elemental changes in the local aerodynamics compared to the isolated case. A comparison of the computed surface pressures for the isolated (Fig. 12(a)) and datum (Fig. 12(b)) receiver configurations at zero incidence illustrates that the diffracted shock path in the multi-body case is in close vicinity to the lifting fins $\left(\Delta \mathrm{x}_{\mathrm{f}} / \mathrm{D}=-0.7\right)$. The parameter $\Delta \mathrm{x}_{\mathrm{f}}$ is defined as the minimum distance between the diffracted shock and the leading edge of the lifting fin at $\phi=90^{\circ}$. The diffracted shock induces a change in the local flow pitch angle close to the lifting fins which the CFD results estimate to be $\Delta \sigma_{\mathrm{p}, \mathrm{up}}=-2.6^{\circ}$. This local flow pitch angle has been extracted from the CFD solution at a single point in the flowfield at the fin mid-span location (midway between the fin root and tip) and a short distance upstream of the fin leading edge $\left(\Delta x_{f} / D=-0.05\right)$. This negative change in flow pitch indicates a 'downwash' effect upstream of the fin. Both inter-fin regions exhibit areas of positive differential induced pressure (where the local pressure is greater than the equivalent isolated case) as a result of the impinging shock front (Fig. 13 (a),(b)). Fig. 13(a) also highlights the effect of the post-shock expansion wave from the generator on the receiver body pressure distribution. Moreover, the negative flow pitch angle leads to an additional region of positive differential pressure on the nearside inter-fin region but an area of relative expansion flow on the farside interfin region $\left(\Phi \approx 80^{\circ}\right)$ which acts to temper the impact of the diffracted shockwave (Fig. 14). This indicates that the strength of the leading edge shock from the lifting fin is modified from the isolated case and this, in turn, modifies the impact of the primary impinging shockwave. In other words, there are two key elemental effects 
which are prominent in the datum interference flowfield, one due to pressure footprints and the other due to flow angularity downstream of the diffracted shock.

In addition to the effect of the shock on the receiver body, the local changes in pressure and flow pitch angle upstream of the fins also affects the fin aerodynamics. A typical first-order estimate ${ }^{33}$ of the normal force acting on a fin of zero thickness at an incidence of $\sigma=-2.6^{\circ}$ is equal to $C_{Z}=-0.055$. Although this agrees reasonably well with the calculated normal force coefficient for one of the lifting fins $\left(\Delta \mathrm{C}_{\mathrm{Z}, \mathrm{fin}} / 2=-0.061\right)$, the induced changes in the fin $\Delta \mathrm{C}_{\mathrm{Z} \text {,fin }}$ are not solely due by the changes in flow pitch angle. A more detailed analysis of the fin pressure distribution reveals that the induced fin loads are a more complex function of more than one element. The upper surface of the lifting fin is indeed dominated by the augmented strength of the leading edge shock as a result of the negative flow pitch ahead of the fin (Fig. 15 (a)). However, the local pressure, especially on the fore panel, is further increased by the impinging shock compression footprint, as noticed in the inter-fin region close to the fin upper surface (Fig. 12). There is little change to the fore panel pressure on the fin lower surface (Fig. 15 (b)). This is unexpected since the negative flow pitch acts to reduce the pressure in this region. However, the diffracted primary shock compression footprint acts to temper the impact of the flow pitch on the lower surface and overall, there is a negligible change in comparison with the isolated pressure distribution.

Overall, it is clear that even for the geometrically simple datum configuration the aerodynamics associated with the body and fin are highly complicated and the observed forces and moments are influenced by several elemental interference effects. However, it is possible to say that the influence of the flow pitch angle on the fin upper surface dominates the normal force interference for the fin $\left(\Delta C_{Z, \text { fin }}=-0.122\right)$. Due to the distance of the fins from the receiver leading edge where the moment reference is located, this results in the induced changes in the fin aerodynamics dominating the overall pitching moment interference load $\left(\Delta \mathrm{C}_{\mathrm{m}, \mathrm{fin}}=0.814\right)$.

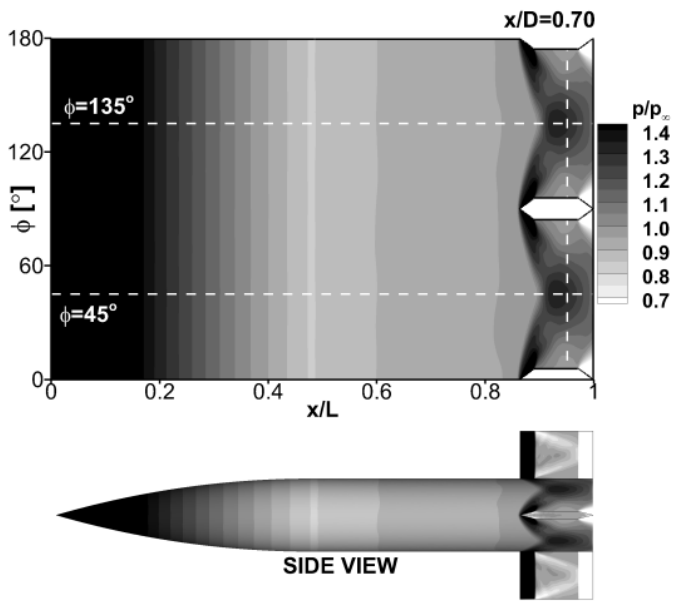

(a) isolated receiver

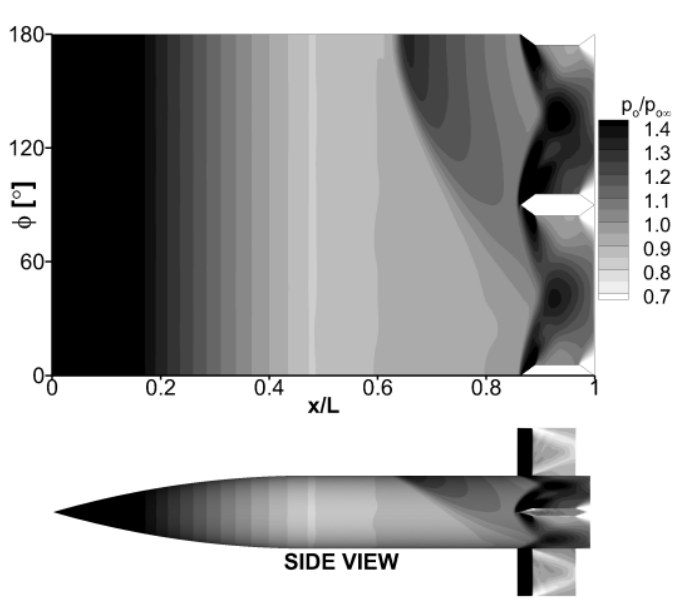

(b) Multibody configuration 
Fig. 12 Calculated receiver surface pressure contours for (a) isolated receiver $\sigma_{R}=0^{\circ}$ and (b) datum multibody configuration $\Delta \mathrm{x}_{\mathrm{w}} / \mathrm{D}=0 \Delta \mathrm{z}_{\mathrm{w}} / \mathrm{D}=2.94, \sigma_{\mathrm{R}}=0^{\circ} \sigma_{\mathrm{G}}=0^{\circ}$.

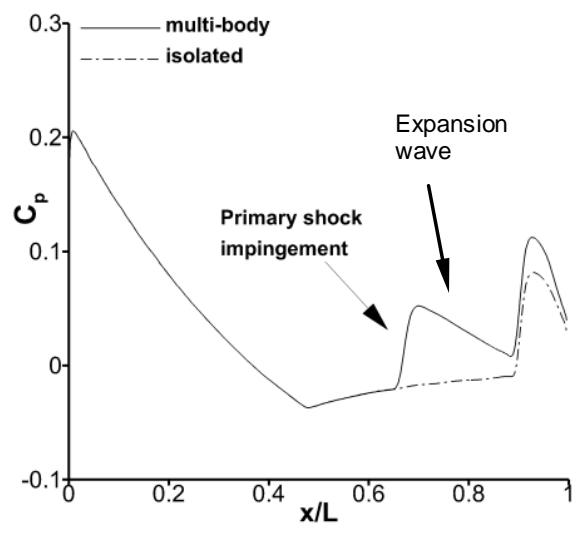

(a) nearside quarter $\phi=135^{\circ}$

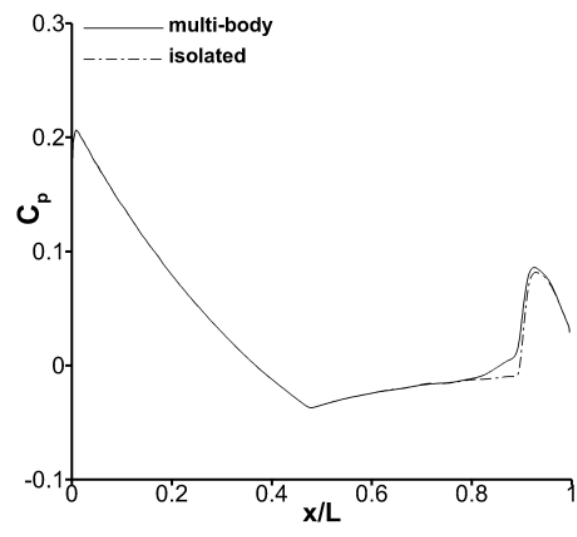

(b) farside quarter $\phi=45^{\circ}$

Fig. 13 Calculated receiver body surface pressure distributions (a-b): for $\Delta x_{w} / D=0 \Delta z_{w} / D=2.94, \sigma_{R}=0^{\circ}$ $\sigma_{\mathrm{G}}=\mathbf{0}^{\circ}$.

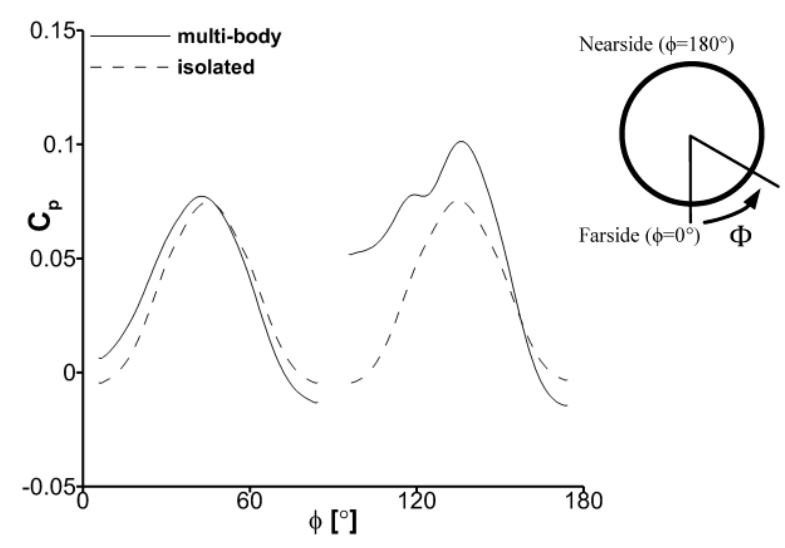

Fig. 14 Calculated receiver surface pressure distribution at $\mathrm{x} / \mathrm{D}=7$ for $\Delta \mathrm{x}_{\mathrm{w}} / \mathrm{D}=0 \Delta \mathrm{z}_{\mathrm{w}} / \mathrm{D}=2.94, \sigma_{\mathrm{R}}=0^{\circ} \sigma_{\mathrm{G}}=0^{\circ}$

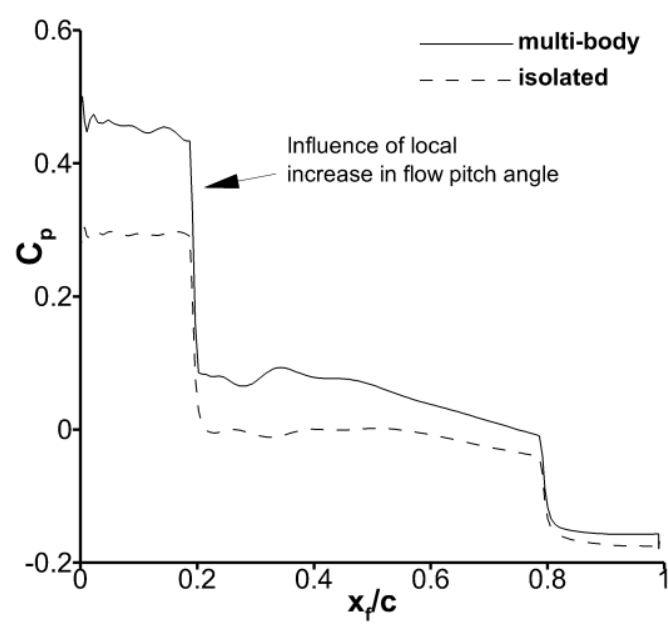

(a) chordwise pressure distribution on lifting fin upper

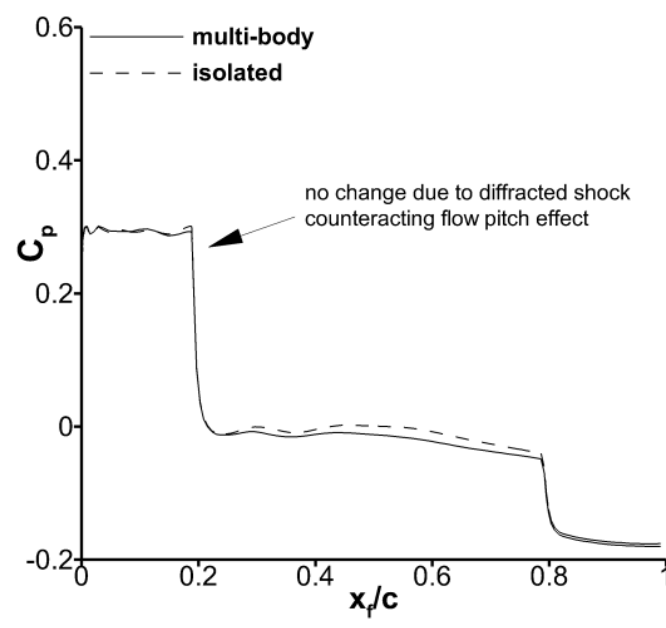

(b) chordwise pressure distribution on lifting fin lower 


\section{Fig. 15 Calculated receiver fin surface pressure distributions at $y_{f} / b=0.5$ for $\Delta x_{w} / D=0 \Delta z_{w} / D=2.94, \sigma_{R}=0^{\circ}$ $\sigma_{G}=0^{\circ}$ (a) upper fin surface (b) lower finer surface.}

\section{Effect of axial stagger on the receiver body at $\sigma_{R}=0^{\circ}$}

Further to the discussion of the datum configuration, the other axial configurations will now be discussed. The measurements show that the relative axial position $\left(\Delta \mathrm{x}_{\mathrm{w}} / \mathrm{D}\right)$ has a notable impact on the normal force and pitching moment of the receiver (Fig. 10). Broadly the interference characteristics are similar for a range of receiver incidence angles and this section investigates a subset in more detail where the receiver body is at zero incidence. The CFD results are used to investigate the underlying aerodynamics for four axial stagger configurations $\left(\Delta \mathrm{x}_{\mathrm{w}} / \mathrm{D}=-1.65,0,1.67,2.68\right)$ for the receiver and sharp generator at $\sigma_{\mathrm{R}}=0^{\circ}$ (Fig. 16).

In these configurations, the generator is placed upstream of the receiver (Fig. 16(a), (b)), axially aligned (Fig. 16(c)) and also downstream of the receiver (Fig. 16(d)). The shadowgraph visualizations show the aftward movement of the primary shock impingement location on the receiver body as the axial stagger changes from $\Delta \mathrm{x}_{\mathrm{w}} / \mathrm{D}=2.68$ through to $\Delta \mathrm{x}_{\mathrm{w}} / \mathrm{D}=-1.65$. Although not evident in the shadographs, it is worth noting that the disturbance flowfield also comprises expansion waves from the generator body. For the most upstream configuration $\left(\Delta \mathrm{x}_{\mathrm{w}} / \mathrm{D}=2.68\right)$, the primary shock impinges onto the receiver towards the end of the forebody section (Fig. 16(a)), while for the aftmost configuration this primary shock impinges just ahead of the fins (Fig. 16(d)). The shock diffracts around the receiver body to some extent in all cases and can still be observed on the farside of the receiver in Fig. 16(a) and (b). The impingement of the primary shock on the receiver nearside results in a reflected shock which reflects back to the generator body. The receiver bow shock strikes the generator body and results in a second reflected shock which returns towards the receiver and impinges at the trailing edge of the uppermost fin. This has a negligible impact on the receiver forces and moments.

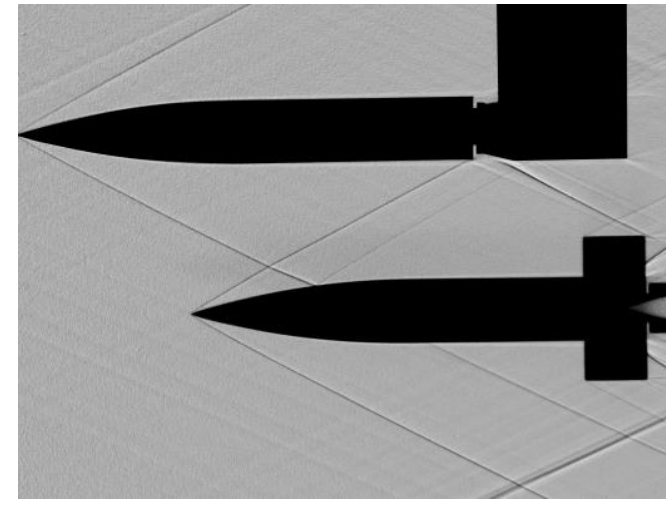

(a) $\Delta x_{w} / D=2.68$

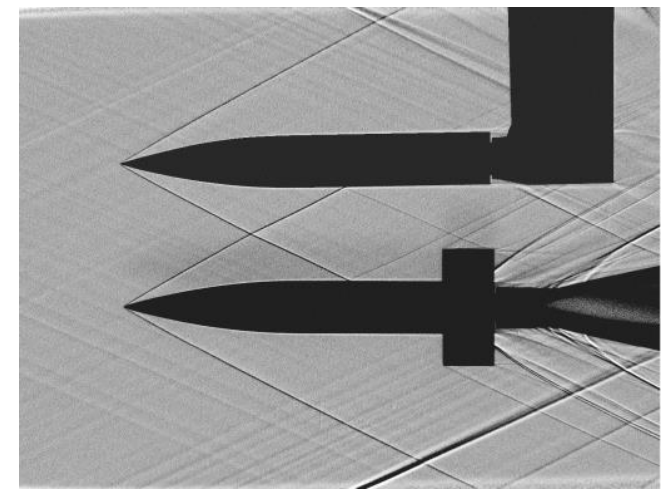

(c) $\Delta \mathrm{x}_{\mathrm{w}} / \mathrm{D}=0$ 


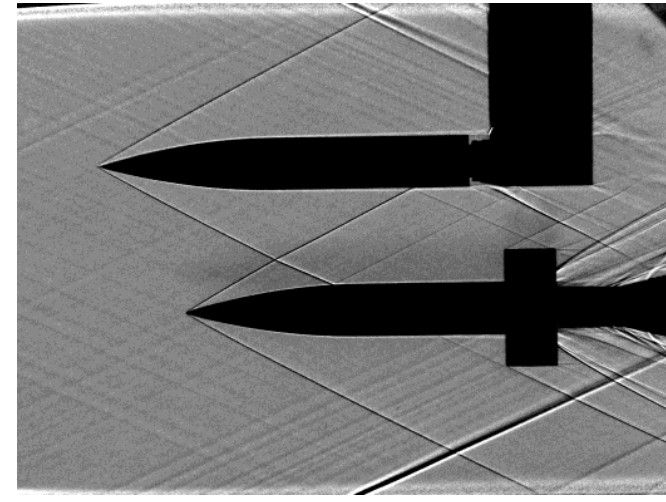

(b) $\Delta \mathrm{x}_{\mathrm{w}} / \mathrm{D}=1.67$

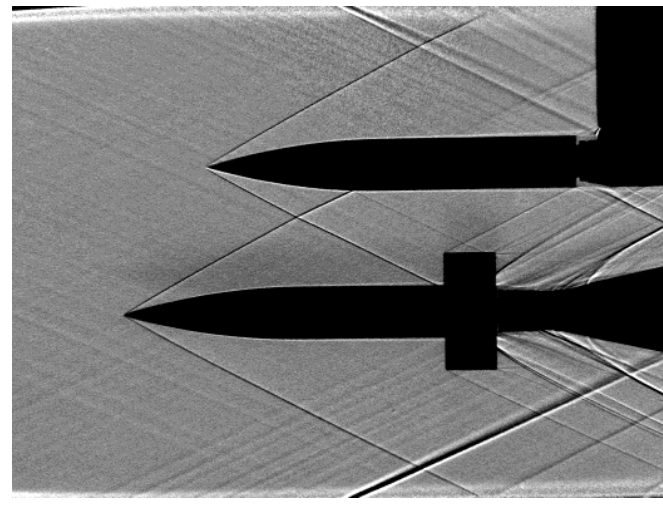

(d) $\Delta \mathrm{x}_{\mathrm{w}} / \mathrm{D}=-1.65$

Fig. 16 Measured shadowgraph visualizations for different axial stagger settings with a fixed lateral spacing $\left(\Delta \mathbf{z}_{\mathrm{w}} / \mathrm{D}=2.94\right)$ and zero body incidence $\left(\sigma_{\mathrm{R}}=0^{\circ} \sigma_{\mathrm{G}}=0^{\circ}\right)$. (a) $\Delta \mathrm{x}_{\mathrm{w}} / \mathrm{D}=2.68$, (b) $\Delta \mathrm{x}_{\mathrm{w}} / \mathrm{D}=1.67$, (c) $\Delta x_{w} / D=0$, (d) $\Delta x_{w} / D=-1.65$.

\section{Forces and moments for the receiver body at $\sigma_{R}=0^{\circ}$}

The interference loads for cases at zero incidence are sensitive to the axial stagger configuration due to the changes in the axial impingement location and proximity of the primary shock to the fins (Fig. 17) and consequently the interference loads tend to be bespoke to each configuration. In general, the sign of the normal force and pitching moment interference loads changes over the shock impingement range considered. For example, the normal force interference is slightly positive when the generator is placed far upstream of the receiver $\left(\Delta \mathrm{x}_{\mathrm{w}} / \mathrm{D}=2.68\right)$ and thus, the receiver body is attracted to the generator body. However, there is also a negative pitching moment which acts to pitch the receiver away from the generator. As the axial impingement location moves aft along the body, the normal force interference becomes more negative, especially for configurations where the shock impinges close to the fins $\left(\Delta \mathrm{x}_{\mathrm{w}} / \mathrm{D}=0\right.$ and $\Delta \mathrm{x}_{\mathrm{w}} / \mathrm{D}=-1.65$ (Fig. 17)). In these configurations, the induced normal force is negative and this acts to translate the receiver away from the generator. The equivalent $\Delta \mathrm{C}_{\mathrm{z}}$ and $\Delta \mathrm{C}_{\mathrm{m}}$ results for the un-finned receiver ${ }^{6}$ are also shown in Fig. 17 to highlight the effect of the fins, especially the notable impact for the datum configuration $\left(\Delta \mathrm{x}_{\mathrm{w}} / \mathrm{D}=0\right)$ where the impinging shock influences the local fin incidence. This effect of the change in local fin incidence is also observed to a lesser extent for the $\Delta \mathrm{x} / \mathrm{D}=1.67$ and $\Delta \mathrm{x} / \mathrm{D}=2.68$ cases and a detailed discussion of the flowfield mechanisms that lead to changes in local fin incidence is given in the next section.

There is a concomitant change in the sign of the pitching moment interference load which changes from negative to positive as the shock impingement location moves aft along the receiver body. In the configuration where the shock impinges at the aft end of the receiver body $\left(\Delta \mathrm{x}_{\mathrm{w}} / \mathrm{D}=-1.65\right)$, the induced change in pitching 
moment is $\Delta \mathrm{C}_{\mathrm{m}}=0.56$ which acts to pitch the nose of the receiver body towards the generator. Such interference characteristics may increase the likelihood of a collision between the bodies. Finally, good agreement is observed between the measured and the calculated interference loads (Fig. 17) and the CFD solutions are used to understand the effect of axial impingement location on the detailed aerodynamic characteristics.

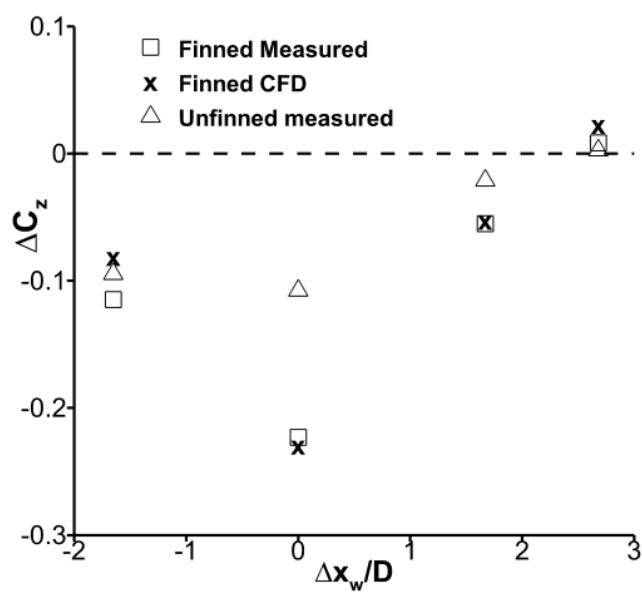

(a)Interference normal force

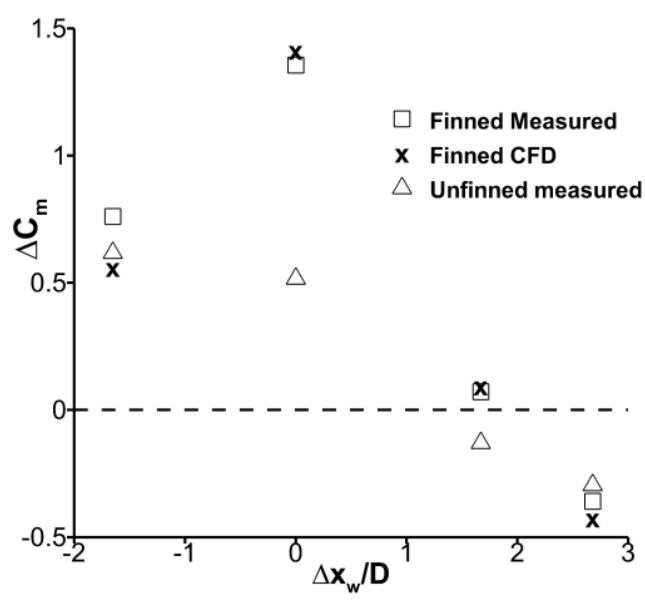

(b)Interference pitching moment

Fig. 17 Receiver interference normal force and pitching moment: sharp generator, $\Delta \mathbf{z}_{\mathrm{w}} / \mathrm{D}=\mathbf{2 . 9 4}$, $\sigma_{\mathrm{R}}=0^{\circ} \sigma_{\mathrm{G}}=0^{\circ}$. Unfinned data from Ref. [6]

\section{Flowfield interpretation for the receiver body at $\sigma_{\mathrm{R}}=0^{\circ}$}

In this section the calculated interference loads are split into the relative contributions from the body of the receiver and that from the stability fins to aid the analysis (Fig. 18, Table 4). As discussed for the datum configuration, the induced change in the flow pitch angle upstream of the lifting fin $\left(\Delta \sigma_{p, u p}\right)$ is an important parameter with respect to the fin interference loads and it is also provided in Table 4.

As the axial stagger is increased from the datum configuration $\left(\Delta \mathrm{x}_{\mathrm{w}} / \mathrm{D}=0\right)$ to the upstream arrangement $\left(\Delta \mathrm{x}_{\mathrm{w}} / \mathrm{D}=2.68\right)$ the primary impingement location is more towards the receiver nose tip and the distance of the shock to the fin leading edge increases about 0.8D to 3.4D (Fig. 16). Consequently, the magnitude of the induced change in the flow pitch angle close to the lifting fin reduces from $-2.6^{\circ}$ to $-0.3^{\circ}$ (Table 4 ) and the effect of the fins on both the overall changes in normal force $\left(\Delta \mathrm{C}_{\mathrm{z}}\right)$ and change in pitching moment $\left(\Delta \mathrm{C}_{\mathrm{m}}\right)$ are relatively small. For this upstream axial stagger configuration $\left(\Delta \mathrm{x}_{\mathrm{w}} / \mathrm{D}=2.68\right)$, the overall interference normal

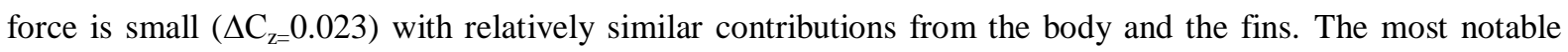
effect at this axial stagger $\left(\Delta \mathrm{x}_{\mathrm{w}} / \mathrm{D}=2.68\right)$ is the induced pitch down moment $\left(\Delta \mathrm{C}_{\mathrm{m}}=-0.43\right)$ which is dominated by aerodynamic changes over the body $\left(\Delta \mathrm{C}_{\mathrm{m}, \mathrm{bod}}=-0.33\right)$. Although the changes in the body normal force are very 
small, the axial pressure distributions over the near and far-side surfaces change, due to the angle of the impinging shock, and there is a consequent change in pitching moment. Broadly for this configuration, the pressure distributions on the fin show very minor changes on the lower surface, with modest reductions in $\mathrm{C}_{\mathrm{p}}$ on the fin upper surface when compared with the isolated receiver configuration (Fig. 19(a) and (b))

When the axial stagger is reduced to $\Delta \mathrm{x}_{\mathrm{w}} / \mathrm{D}=1.67$ (Fig. 16(b)), the induced flow pitch angle at the fin increases in magnitude to $\Delta \sigma_{p, u p}=-1.2^{\circ}$ (Table 4) as the diffracted primary impinging shock is now closer to the fin leading edge. Nevertheless, the interference normal force is small (Fig. 17(a)) with only modest contributions from both the receiver body and the fins (Table 4). This is because the effect of the shock impingement on the receiver nearside is approximately balanced by the impact of the generator expansion wave on the nearside of the receiver as well as the effect of the diffracted shock on the farside of the receiver ${ }^{6}$. For this case, the changes in the pressure coefficient distributions along the fin center-span are small with the only difference being a slight increase in $C_{p}$ on the front panel on the upper surface (Fig. 19(a)). The combined effect of the changes in pressure and flow pitch angle over the fins is that the modest increase in pitching moment from the fins $\left(\Delta \mathrm{C}_{\mathrm{m}, \text { fins }}=0.21\right)$ is offset by the induced pitch down contribution from the body $\left(\Delta \mathrm{C}_{\mathrm{m}, \text { body }}=-0.12\right)$ which gives an overall marginal pitch up change of $\Delta \mathrm{C}_{\mathrm{m}}=0.094$ (Table 4).

Finally, for the case where the generator is located downstream of the receiver $\left(\Delta x_{w} / D=-1.65\right)$, the receiver body is mainly influenced by the compression footprint of the impinging shock which impacts close to the base (Fig. 16 (d)). Moreover, the fin interference loads are also caused by this compression footprint since there is no upstream flow pitch change ahead of the fins $\left(\Delta \sigma_{p, u p}=0.0^{\circ}\right)$ as the shock impacts on the fin upper surface. Overall there is a notable induced positive pitching moment $\left(\Delta \mathrm{C}_{\mathrm{m}}=0.559\right)$ which is strongly dominated by the changes on the receiver centrebody.

Overall, the dominant interference mechanism which acts on the lifting fin changes as a function of the axial impingement location. Analysis of the datum configuration has shown that the fin interference loads are a complex function of the pressure footprints from the compression and expansion waves as well as the upstream flow pitch induced by the shock. The potency of these three individual mechanisms vary as the generator is moved forward or aft from the datum configuration and the overall fin interference loads are frequently influenced by all three. However, in general the fin loads are dominated by the generator forebody expansion waves when the generator is furthest upstream of the receiver with an axial stagger of $\Delta \mathrm{x}_{\mathrm{w}} / \mathrm{D}=2.68$ (Fig. 19 (a)). The dominant mechanism changes to the local flow pitch when the generator is located at $\Delta \mathrm{x}_{\mathrm{w}} / \mathrm{D}=1.67$ and $\Delta \mathrm{x}_{\mathrm{w}} / \mathrm{D}=0$ as a result of the proximity of the diffracted shock to the fin leading edge. Evidence of this flow pitch 
effect is also seen in the circumferential pressure distribution (Fig. 20) in the inter-fin region at $\mathrm{x} / \mathrm{D}=7$, where a small region of negative differential pressure is observed close to the lower fin surface. Finally, the compression footprint of the generator bow shockwave dominates the fin interference loads when the generator is aft of the receiver $\left(\Delta \mathrm{x}_{\mathrm{w}} / \mathrm{D}=-1.65\right)$, although the footprint extent is small and the fin interference loads are limited (Table 4).

Table 4 Tabulated component breakdown of the calculated receiver interference loads for different axial stagger settings: sharp generator, $\Delta \mathrm{z}_{\mathrm{w}} / \mathrm{D}=2.94, \sigma_{\mathrm{R}}=0^{\circ} \sigma_{\mathrm{G}}=0^{\circ}$

\begin{tabular}{cc|ccc|ccc}
\hline $\boldsymbol{\Delta} \mathbf{x}_{\mathrm{w}} / \mathrm{D}$ & $\boldsymbol{\Delta} \boldsymbol{\sigma}_{\mathrm{p}, \text { up }}\left[^{\circ}\right]$ & $\boldsymbol{\Delta} \mathrm{C}_{\mathrm{z}, \text { bod }}$ & $\boldsymbol{\Delta} \mathrm{C}_{\mathrm{z}, \text { fins }}$ & $\boldsymbol{\Delta} \mathrm{C}_{\mathrm{z}}$ & $\boldsymbol{\Delta} \mathrm{C}_{\mathrm{m}, \text { bod }}$ & $\boldsymbol{\Delta} \mathrm{C}_{\mathrm{m}, \text { fins }}$ & $\boldsymbol{\Delta} \mathrm{C}_{\mathrm{m}}$ \\
\hline $\mathbf{- 1 . 6 5}$ & 0.0 & -0.071 & -0.010 & -0.081 & 0.484 & 0.074 & 0.559 \\
$\mathbf{0}$ & -2.6 & -0.108 & -0.122 & -0.23 & 0.598 & 0.814 & 1.412 \\
$\mathbf{1 . 6 7}$ & -1.2 & -0.020 & -0.033 & -0.053 & -0.120 & 0.213 & 0.094 \\
$\mathbf{2 . 6 8}$ & -0.3 & 0.009 & 0.013 & 0.023 & -0.330 & -0.096 & -0.426 \\
\hline
\end{tabular}
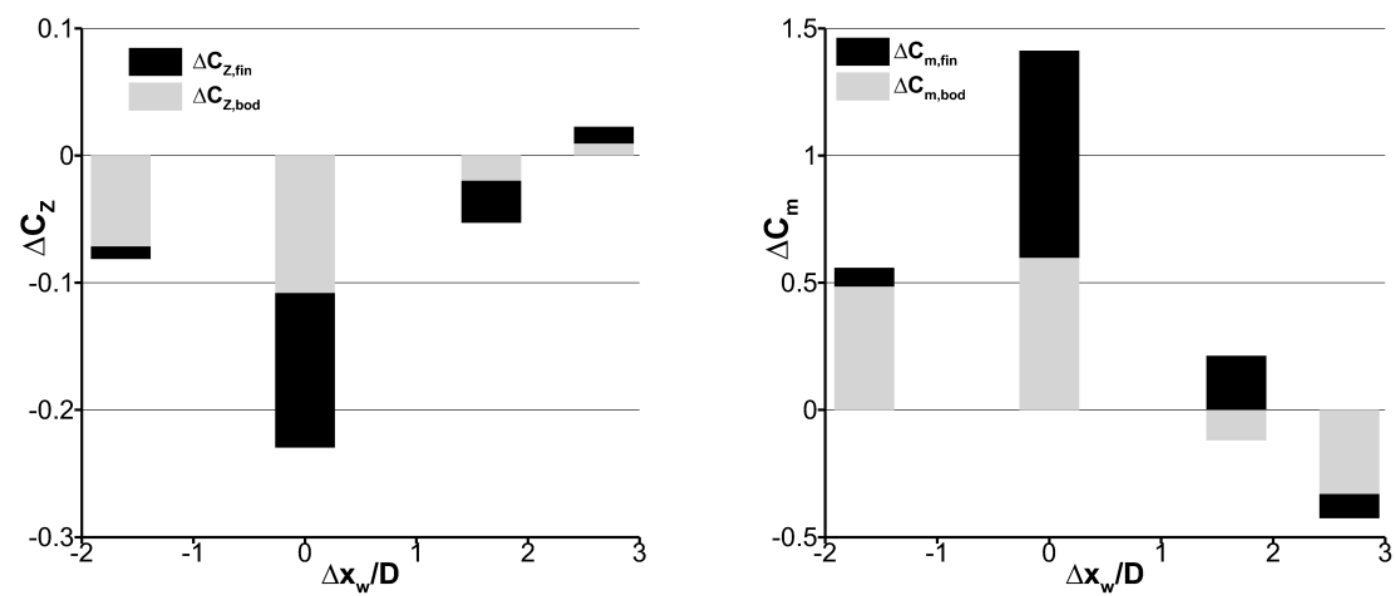

Fig. 18 Breakdown of the calculated receiver normal force and pitching moment interference loads for $\Delta x_{w} / D=-1.65,0,1.67,2.68$ : sharp generator, $\Delta z_{w} / D=2.94, \sigma_{R}=0^{\circ} \sigma_{G}=0^{\circ}$ 


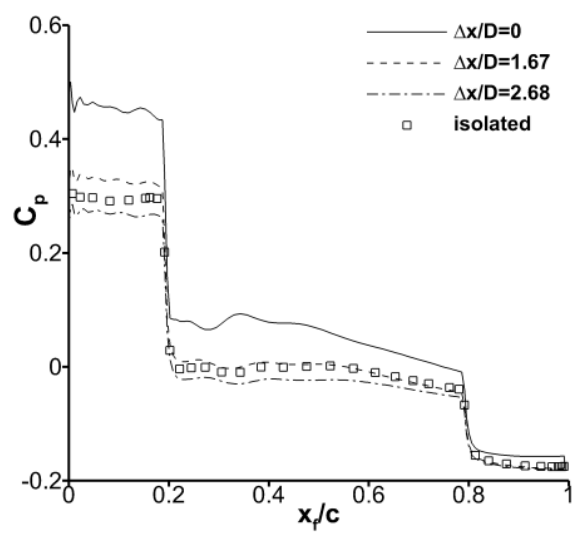

(a) chordwise pressure distribution on fin upper surface

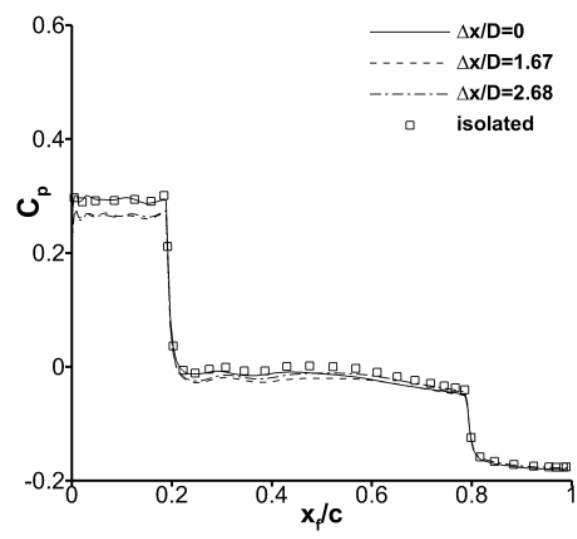

(b) chordwise pressure distribution on fin lower surface

Fig. 19 Calculated receiver fin surface pressure distributions at $y_{\mathrm{f}} / \mathbf{b}=\mathbf{0 . 5}$ for different axial stagger settings using the sharp generator, $\Delta \mathbf{z}_{\mathrm{w}} / \mathrm{D}=\mathbf{2 . 9 4}, \sigma_{\mathrm{R}}=0^{\circ} \sigma_{\mathrm{G}}=0^{\circ}$ (a) fin upper surface (b) fin lower surface.

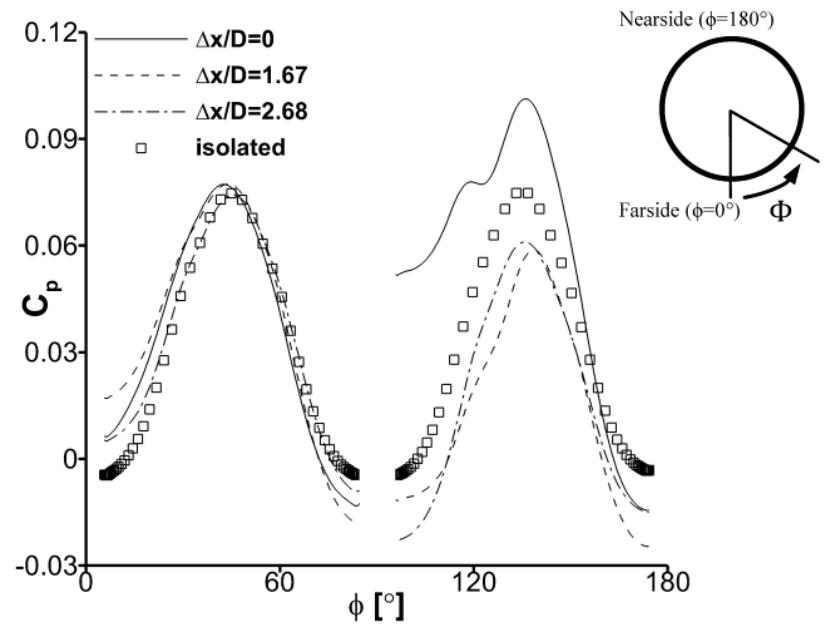

Fig. 20 Calculated receiver body circumferential surface pressure distribution at $\mathrm{x} / \mathrm{D}=7 \mathrm{using}$ a sharp generator, $\Delta \mathbf{x}_{\mathrm{w}} / \mathrm{D}=\mathbf{0} \Delta \mathrm{z}_{\mathrm{w}} / \mathrm{D}=\mathbf{2 . 9 4}, \sigma_{\mathrm{R}}=\mathbf{0}^{\circ} \sigma_{\mathrm{G}}=0^{\circ}$

\section{Effect of receiver incidence}

As the receiver incidence increases from zero, there is a change in the underlying aerodynamics for the isolated receiver where the effects of flow separations, body vortices and streamwise load distribution become more significant. Furthermore, for multi-body configurations, there is a change to the location and strength of the impinging disturbances from the shock and expansion waves and therefore the induced interference flowfield is modified. Consequently, as both the isolated receiver aerodynamics and the impact of the disturbance flowfield are notably different, it is expected that the interference loads will also depend on the receiver incidence setting. This section assesses the measured and predicted data for a subset of the test matrix at one axial stagger $\left(\Delta \mathrm{x}_{\mathrm{w}} / \mathrm{D}=1.67\right)$ to highlight the effect of body incidence on the interference aerodynamics. 


\section{The effect of receiver incidence on the interference forces and moments}

As the receiver incidence increases from $\sigma_{R}=-15^{\circ}$ to $\sigma_{R}=15^{\circ}$, the measured normal force interference load $\left(\Delta \mathrm{C}_{\mathrm{z}}\right)$ becomes more negative and changes from -0.03 to -0.18 (Fig. 21). There is a concomitant increase in the induced pitching moment coefficient and the axial force interference is relatively small. The trends of both normal force and pitching moment interference are broadly monotonic over the incidence range considered. Moreover, good agreement is observed between the measured and calculated interference loads and this demonstrates that the computational method is capable of calculating the interference effects for the receiver at non-zero incidence. The largest magnitude of the measured interference loads are observed at high positive incidence where the receiver is closest to the generator. The aerodynamic characteristics associated with the variation in incidence are investigated in the next section using calculations of the configurations across the range at $\sigma_{R}=-15,-8,0,8,15^{\circ}$.

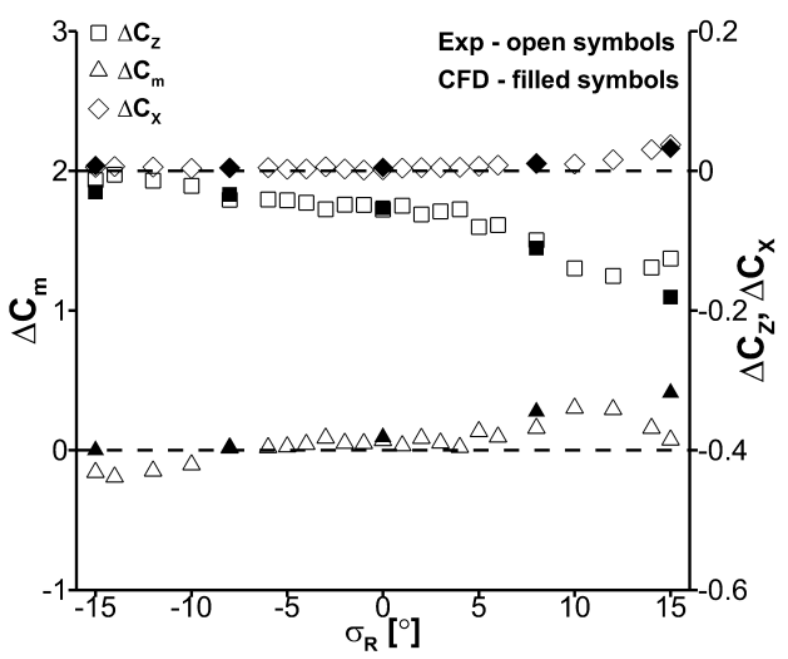

Fig. 21 Effect of receiver incidence on the receiver body interference loads, $\Delta x_{w} / D=1.67 \Delta z_{w} / D=2.94$, $\sigma_{\mathrm{G}}=\mathbf{0}^{\circ}$.

\section{Center of pressure location as a function of incidence}

The induced movement of the longitudinal center of pressure $\left(X_{\mathrm{cp}}\right)$ is an important aspect of the interference characteristics for the marginally stable receiver body. The disturbance flowfield induced by the sharp generator translates the center of pressure on the receiver by up to two calibers over the incidence range considered (Fig. 23). As a result, the receiver becomes statically unstable for $\sigma_{R}=-2^{\circ}$. Previous observations ${ }^{6}$ also indicate, for an unfinned receiver, that the movement of the center of pressure movement is greater if a blunt generator is used as it produces a stronger impinging shock (Fig. 22). For the finned receiver, this change in the disturbance field caused by the blunt generator also amplifies the impact on the static stability margin so that it becomes unstable 
when $\sigma_{R}=-6^{\circ}$ and, consequently, the finned receiver is statically unstable for over about a third of the measured negative incidence range.
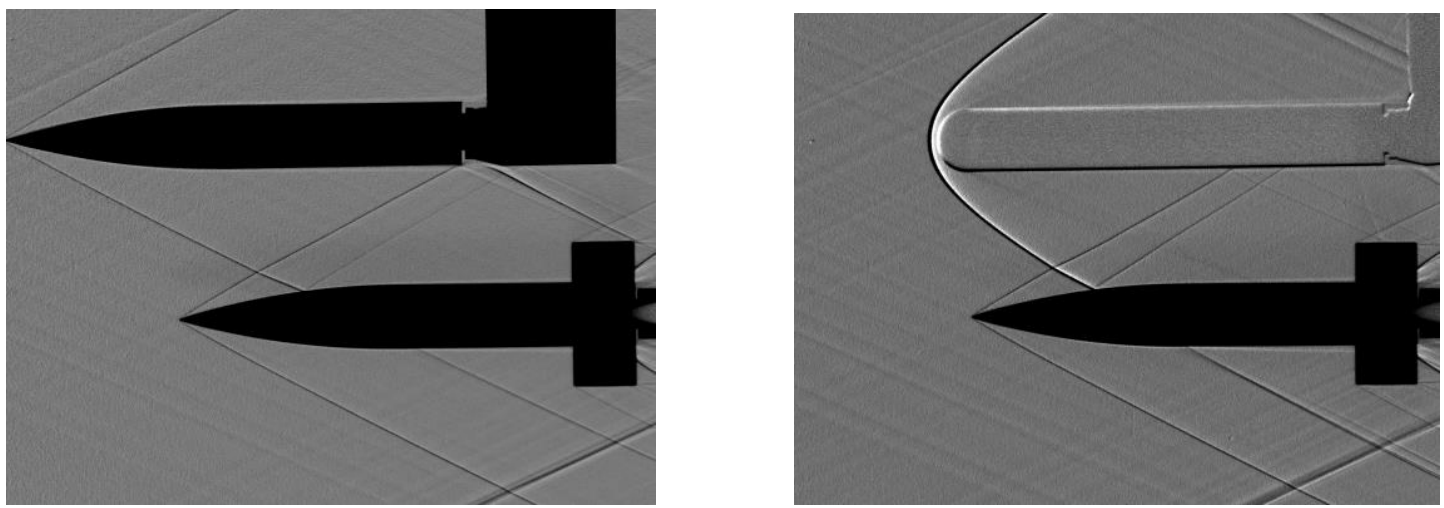

Fig. 22 Comparison of shadowgraphs for the sharp and blunt generators with $\Delta \mathbf{z}_{\mathrm{w}} / \mathrm{D}=\mathbf{2 . 9 4 ,} \sigma_{\mathrm{G}}=0^{\circ} \sigma_{\mathrm{R}}=0$ (a) sharp generator and receiver body: $\Delta \mathbf{x}_{w} / D=2.68$ (b) blunt generator and receiver body: $\Delta \mathbf{x}_{\mathrm{w}} / \mathrm{D}=\mathbf{0 . 4 4}$.

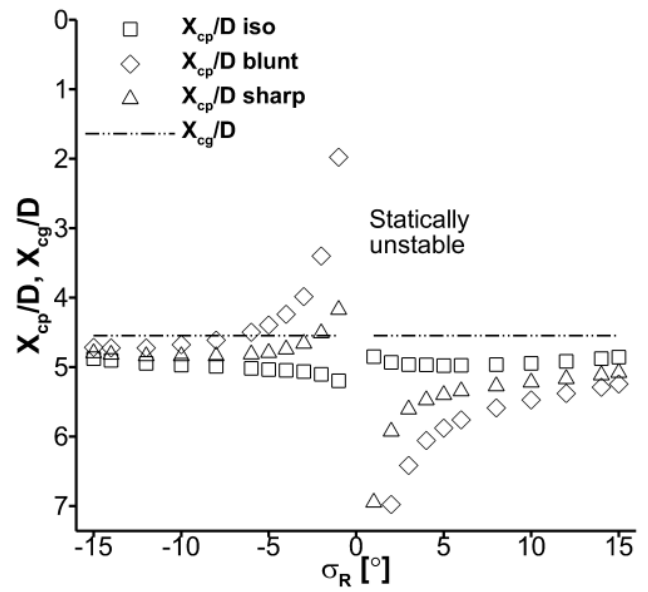

Fig. 23 Effect of receiver incidence on the measured $X_{c p}$ using data from both the sharp and blunt generator configurations: $\Delta \mathbf{z}_{\mathrm{w}} / \mathrm{D}=\mathbf{2 . 9 4}, \sigma_{\mathrm{G}}=0^{\circ}$

\section{The effect of receiver incidence on the interference flowfield}

The local flow conditions in the disturbance flowfield are expected to strongly influence the induced loads on the receiver body. The computed flow solutions for the finned receiver at $\sigma_{R}=-15,-8,0,8,15^{\circ}$ (Fig. 21) and an axial stagger of $\Delta x_{w} / D=1.67$ are used in this section to understand the relationship between the interference loads and the receiver incidence.

Within this subset of configurations, good agreement is observed between the measured and calculated overall forces and moments. The PSP measurements are now compared in Fig. 24 to assess the suitability of the CFD to predict the body surface pressures in terms of the size and location of the interference footprints ${ }^{6}$. Full details of the PSP set-up and method can be found in Chaplin et $\mathrm{al}^{23}$. 


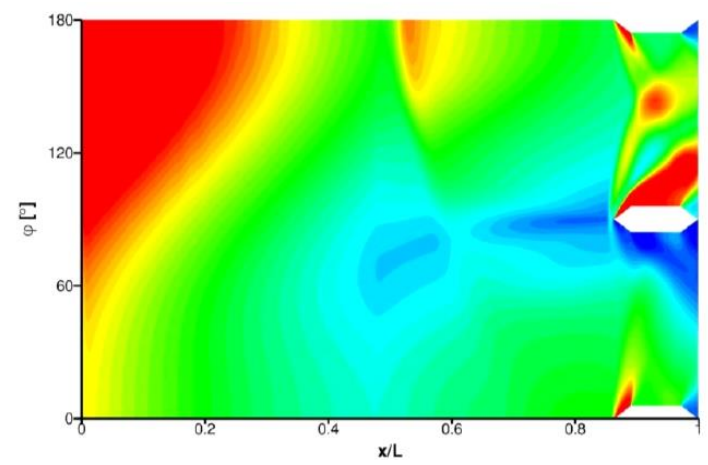

(a) $\sigma_{R}=-8^{\circ} \mathrm{CFD}$

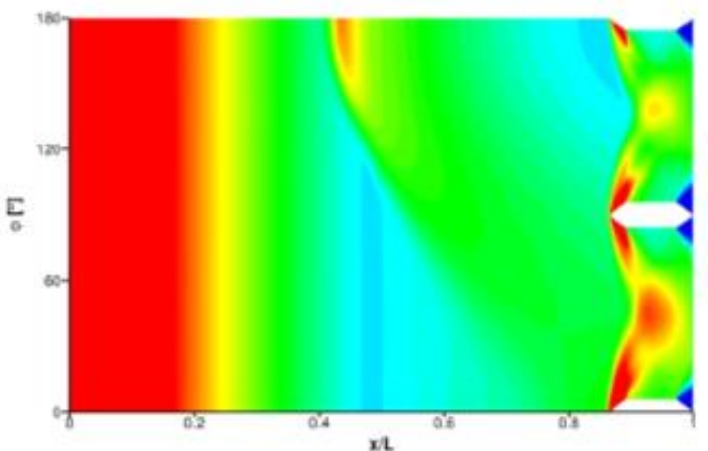

(b) $\sigma_{\mathrm{R}}=0^{\circ} \mathrm{CFD}$

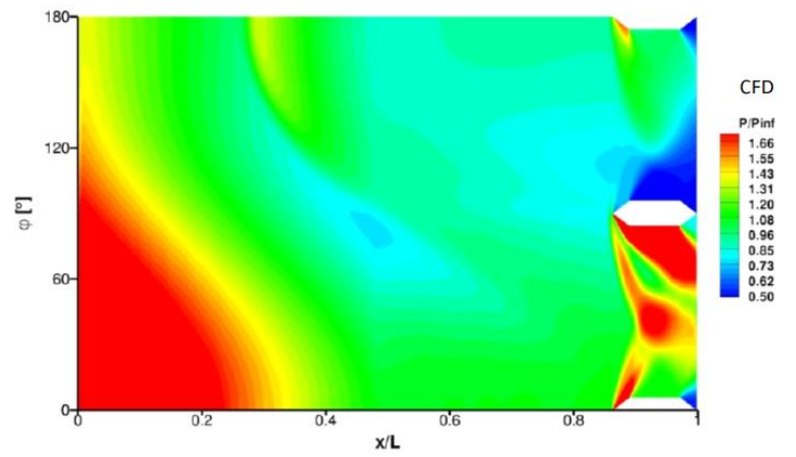

(c) $\sigma_{\mathrm{R}}=8^{\circ} \mathrm{CFD}$

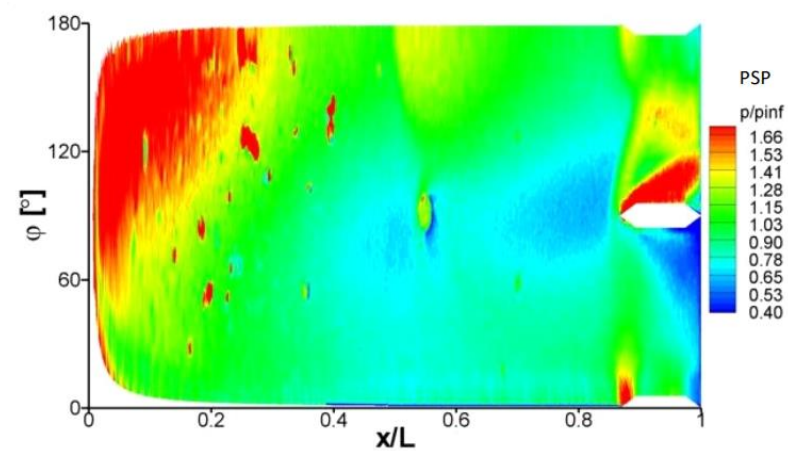

$\sigma_{R}=-8^{\circ} \mathrm{PSP}$

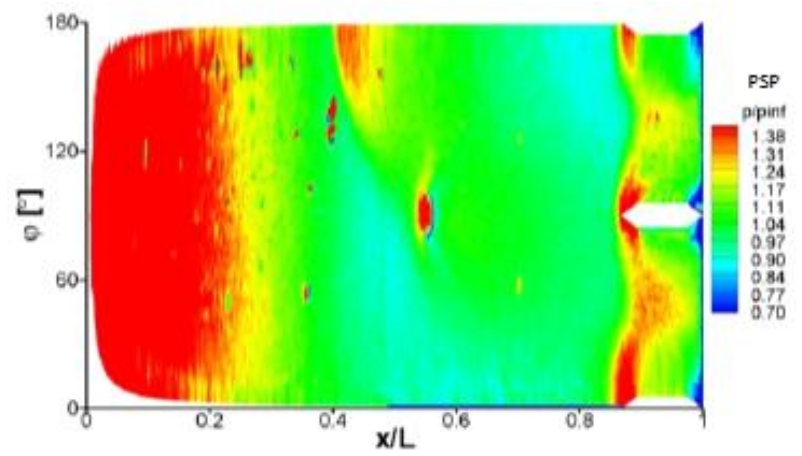

$\sigma_{\mathrm{R}}=0^{\circ} \mathrm{PSP}$

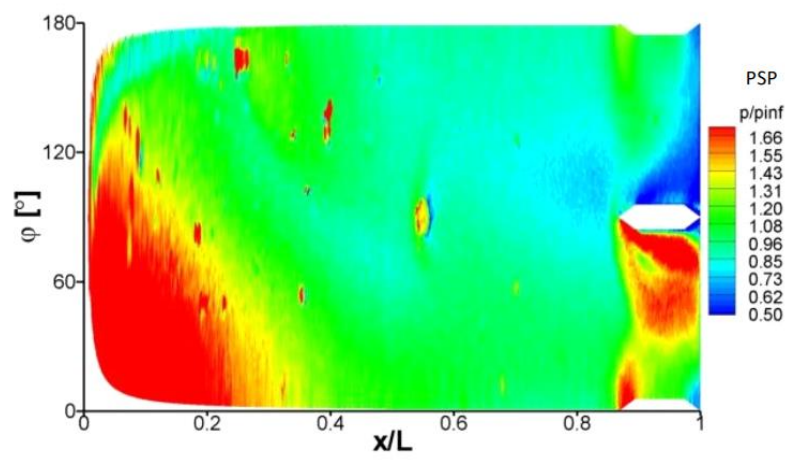

$\sigma_{\mathrm{R}}=8^{\circ} \mathrm{PSP}$

Fig. 24 Comparison of measured (PSP) and predicted (CFD) surface pressures on finned receiver for different receiver incidence settings (a-c): sharp generator, $\Delta x_{w} / D=1.67 \Delta z_{w} / D=2.94, \sigma_{G}=0^{\circ}$

For the selection of incidence configurations shown in Fig. 24, the measured and predicted surface pressures, in particular the interference footprints due to the impinging shockwave and expansion waves are predicted well by the CFD in terms of both magnitude and location on the receiver body. This good agreement is observed for all of the different incidence configurations tested which are not shown here but can be seen in Chaplin ${ }^{6}$. The detailed aerodynamics of the configurations shown in Fig. 24will be discussed below, but the comparison shows that the measured interference flow physics is well captured by the CFD and these predictions can be used to further investigate the interference aerodynamics of other configurations in this paper. 
The previous section has shown that for some configurations the local fin loads can be the dominant factor in the overall interference effects. The underlying flow physics of the fin interference loads for the receiver configurations at incidence are highly complex. Of main interest are three interference mechanisms which combine in different ways as the receiver incidence changes. The mechanisms include the compression footprint from the impinging primary shock, the expansion footprint originating from by the generator forebody and the flow angularity across the impinging primary shock.

When the receiver incidence is zero (Fig. 25 (c)), the fin interference loads are the largest contributor to the overall interference effects (Table 5). The fin interference loads are dominated by the flow pitch upstream of the lifting fins $\left(\sigma_{\mathrm{p}, \text { up }}=-1.2^{\circ}\right)$ which causes elevated pressures on the fore panel of the fin upper surface (Fig. 26 (a)). The upper surface is also affected to a smaller extent by the expansion wave which emanates from the generator forebody Fig. 26 (a),(b)). This effect of flow pitch is the primary reason why the normal force across the range of incidence angles of $-6 \leq \sigma_{R} \leq 6^{\circ}$ is generally negative.

As the receiver incidence becomes more negative (e.g. $\sigma_{R}=-8^{\circ},-15^{\circ}$, the fins are located closer to the generator and are in a stronger part of the disturbance flowfield (Fig. 25 (d), (e)). The generator expansion waves have a significant impact on the local pressures on the upper surface of the fins. For example, at $\sigma_{R}=-8^{\circ}$ the fin is influenced by a moderate flow pitch $\left(\sigma_{\mathrm{p}, \text { up }}=-1.3^{\circ}\right)$ as seen in the pressure distribution on the fin lower surface (Fig. 27 (b)). However, the expansion waves are strong enough to cancel out the expected complimentary pressure rise on the fin upper surface (Fig. 27 (a)) and there is almost no overall fin interference load for this configuration (Table 5). Since it is close to the generator, the receiver body is more strongly affected by the impinging shockwave compared with the low incidence cases and there is also less of an influence of the diffracted shock on the receiver farside ${ }^{6}$. As a result, the body normal force is more negative and the overall normal force interference load is negative $\left(\Delta \mathrm{C}_{Z}=-0.034\right)$. As the incidence reduces further $\left(\sigma_{R}=-\right.$ $15^{\circ}$ ), the fins move closer to the generator and the fin loads are further dominated by a stronger expansion field. In this case the induced changes in pitching moment from the body and from the fins are of the same magnitude, but opposite sign, so that the net effect is zero.

Finally, as the incidence increases from zero to $\sigma_{R}=15^{\circ}$, the diffracted compression shock impinges further towards the receiver forebody and, because of the obliqueness angle of the shock, it also moves closer to the fin lower surface (Fig. 25 (a),(b)). The predicted flow pitch at $\sigma_{R}=8^{\circ}\left(\sigma_{\mathrm{p} \text {,up }}=-2.1^{\circ}\right)$ is larger than the configuration at zero incidence $\left(\sigma_{\mathrm{p} \text {,up }}=-1.2^{\circ}\right)$ yet the fin normal force interference is approximately the same. The reason for this is due to the influence of the diffracted shockwave on the lower surface of the fin (Fig. 28 (a)). The upper 
surface shows the expected pressure rise due to the flow pitch (Fig. 28 (a)) but the compression footprint from the diffracted shock tempers the reduction in pressure on the lower surface as a result of the flow pitch. When the incidence increases further to $\sigma_{R}=15^{\circ}$, the compression footprint augments the lower fin surface more and the fin loads become positive.

The underlying flow physics of the finned receiver becomes increasingly complex when the receiver is placed at incidence. Firstly, the receiver incidence has a subtle yet significant impact on the aerodynamic characteristics. This is due to both the location of the receiver body in the disturbance flowfield as well as the proximity of the fins to the generator body. Secondly, the interference effects can change the stability of the finned receiver to be statically unstable. This is the case for a notable part of the negative incidence range considered when the blunt generator is used (Fig. 23)

Table 5 Tabulated component breakdown of the calculated receiver interference loads for different receiver incidence settings: sharp generator, $\Delta x_{w} / D=1.67 \Delta z_{w} / D=2.94, \sigma_{G}=0^{\circ}$

\begin{tabular}{cc|ccc|ccc}
\hline $\boldsymbol{\sigma}_{\mathrm{R}}\left[^{\circ}\right]$ & $\boldsymbol{\Delta} \boldsymbol{\sigma}_{\mathrm{p}, \text { up }}\left[^{\circ}\right]$ & $\boldsymbol{\Delta} \mathrm{C}_{\mathrm{z}, \text { bod }}$ & $\boldsymbol{\Delta} \mathrm{C}_{\mathrm{z} \text {,fin }}$ & $\boldsymbol{\Delta} \mathrm{C}_{\mathrm{z}}$ & $\boldsymbol{\Delta} \mathrm{C}_{\mathrm{m}, \text { bod }}$ & $\boldsymbol{\Delta} \mathrm{C}_{\mathrm{m}, \text { fin }}$ & $\boldsymbol{\Delta} \mathrm{C}_{\mathrm{m}}$ \\
\hline $\mathbf{- 1 5}$ & -0.3 & -0.063 & 0.033 & -0.030 & 0.236 & -0.235 & 0.001 \\
$\mathbf{- 8}$ & -1.3 & -0.033 & -0.001 & -0.034 & 0.024 & -0.008 & 0.016 \\
$\mathbf{0}$ & -1.2 & -0.020 & -0.033 & -0.053 & -0.120 & 0.213 & 0.094 \\
$\mathbf{8}$ & -2.1 & -0.076 & -0.035 & -0.111 & 0.051 & 0.225 & 0.277 \\
$\mathbf{1 5}$ & -1.2 & -0.192 & 0.011 & -0.181 & 0.484 & -0.071 & 0.412 \\
\hline
\end{tabular}




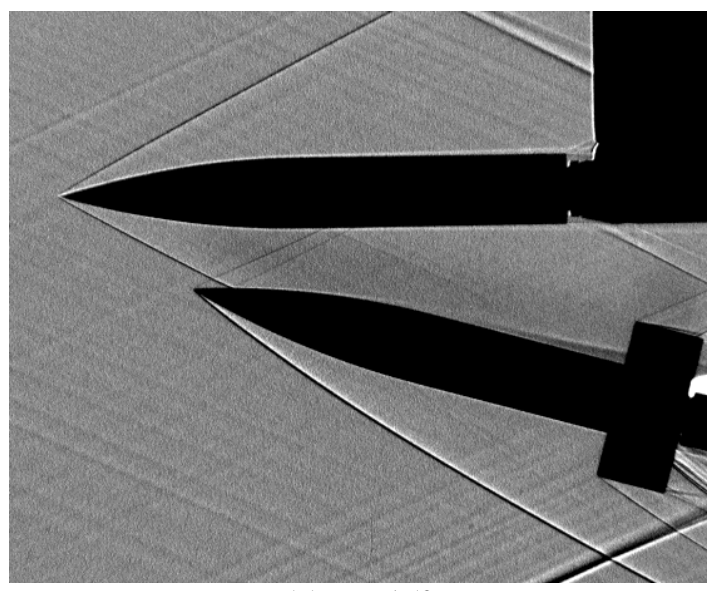

(a) $\sigma_{R}=15^{\circ}$

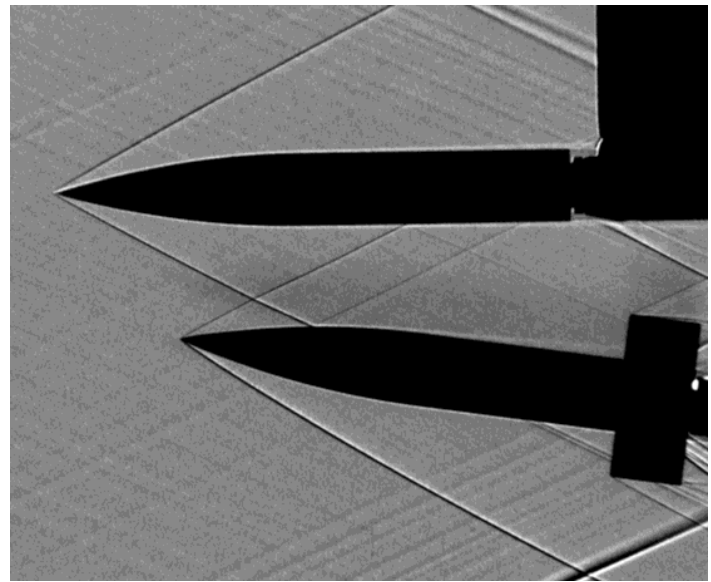

(b) $\sigma_{\mathrm{R}}=8^{\circ}$

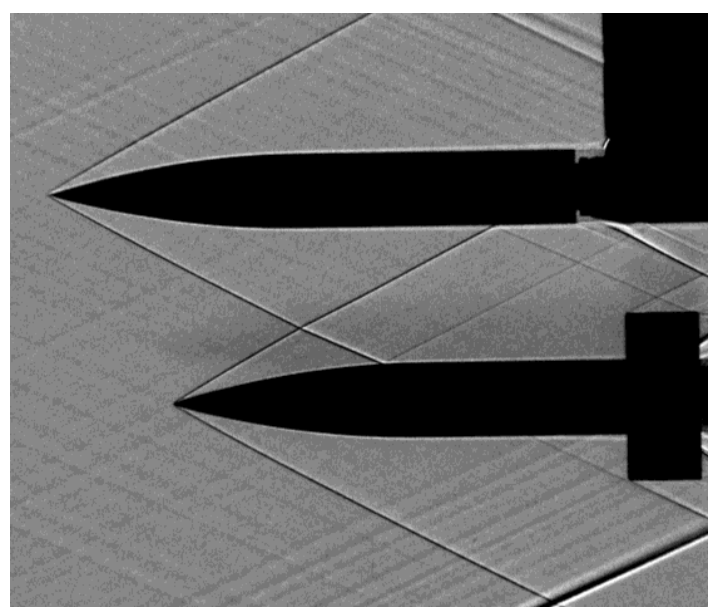

(c) $\sigma_{R}=0^{\circ}$

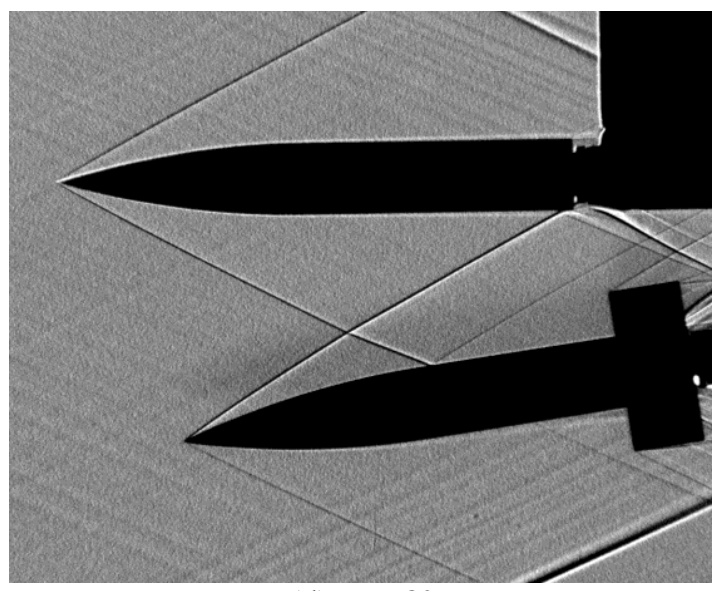

(d) $\sigma_{R}=-8^{\circ}$

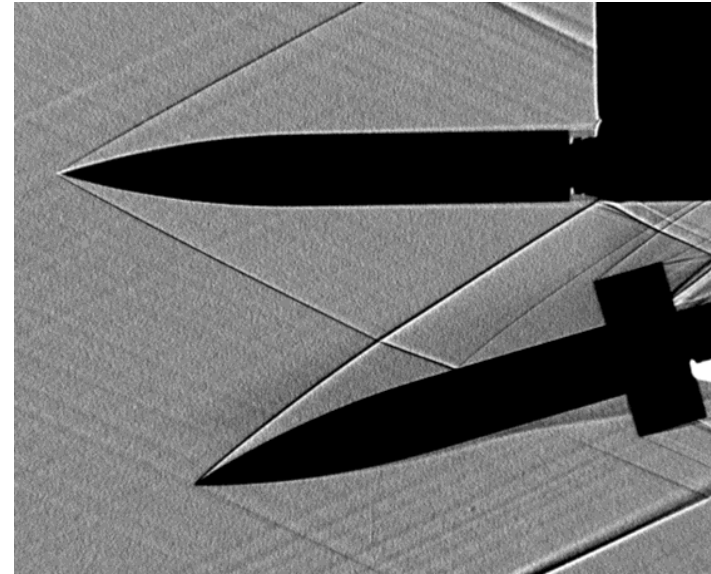

(e) $\sigma_{R}=-15^{\circ}$

Fig. 25 Measured shadowgraph visualizations for different receiver incidence settings (a-e): sharp generator, $\Delta \mathbf{x}_{\mathrm{w}} / \mathrm{D}=\mathbf{1 . 6 7} \Delta \mathrm{z}_{\mathrm{w}} / \mathrm{D}=\mathbf{2 . 9 4}, \sigma_{\mathrm{G}}=0^{\circ}$ 


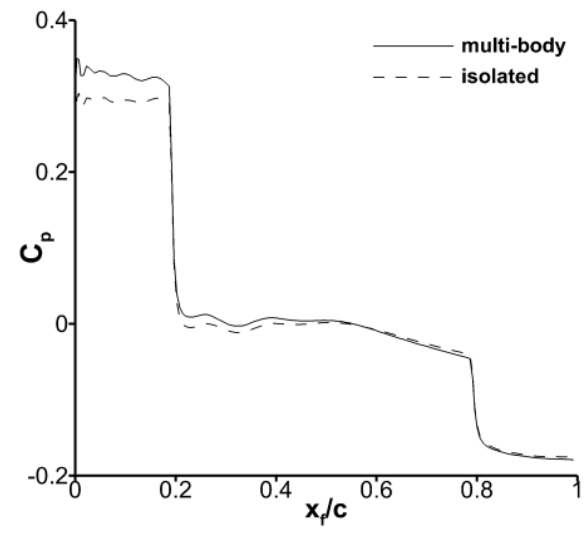

(a) chordwise pressure distribution on fin upper surface

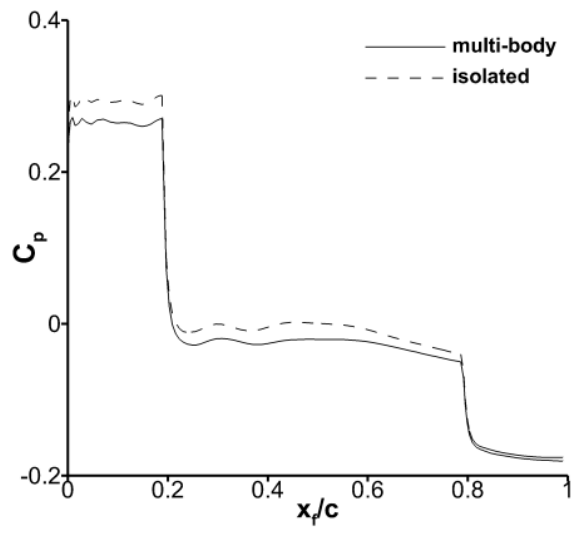

(b) chordwise pressure distribution on fin lower surface

Fig. 26 Calculated receiver fin surface pressure distributions at $y_{\mathrm{f}} / \mathrm{b}=\mathbf{0 . 5}$ : $\operatorname{sharp}$ generator, $\Delta \mathbf{z}_{\mathrm{w}} / \mathrm{D}=2.94, \sigma_{\mathrm{R}}=0^{\circ}$ $\sigma_{\mathrm{G}}=0^{\circ}(\mathrm{a})$ upper surface (b) lower surface.

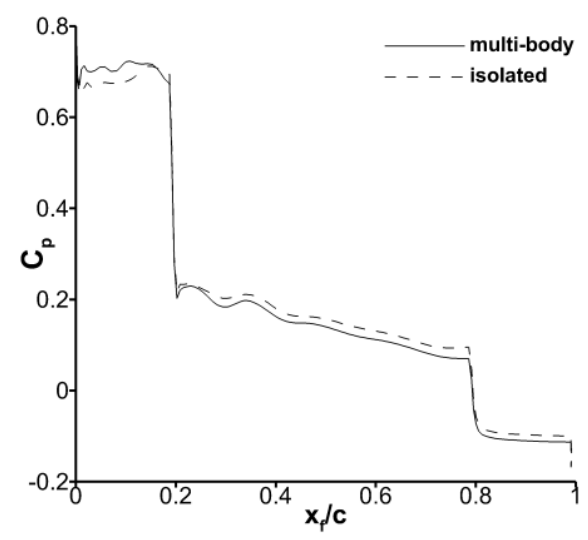

(a) chordwise pressure distribution on fin upper surface

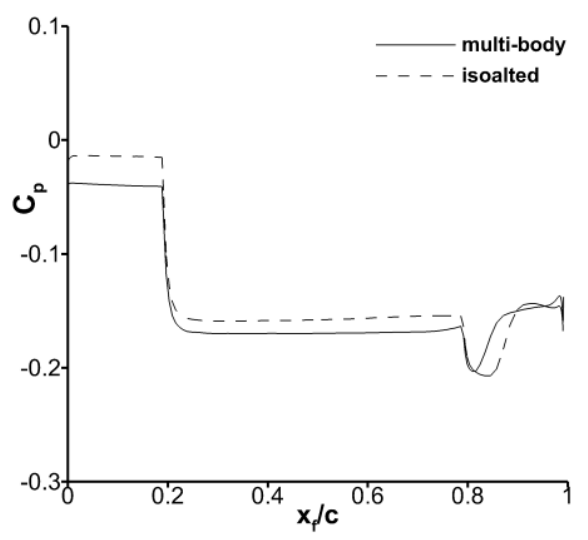

(b) chordwise pressure distribution on fin lower surface

Fig. 27 Calculated receiver fin surface pressure distributions at $y_{\mathrm{f}} / \mathrm{b}=\mathbf{0 . 5}$ : sharp generator, $\Delta \mathbf{z}_{\mathrm{w}} / \mathrm{D}=\mathbf{2 . 9 4}, \sigma_{\mathrm{R}}=-8^{\circ}$ $\sigma_{G}=0^{\circ}$, (a) upper surface (b) lower surface. Note the different $y$-axis scales. 


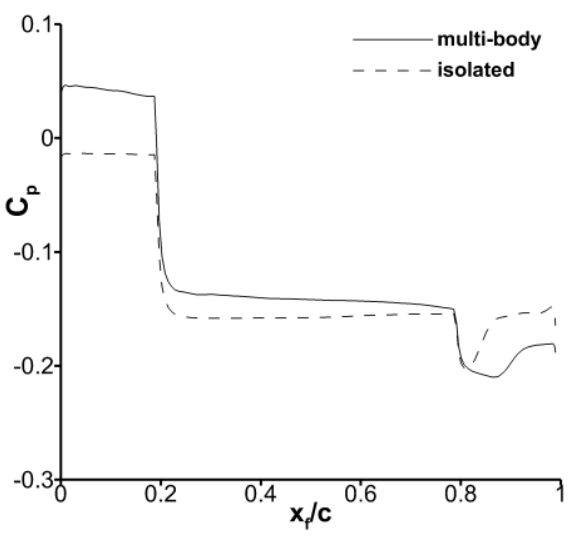

(a) chordwise pressure distribution on fin upper surface

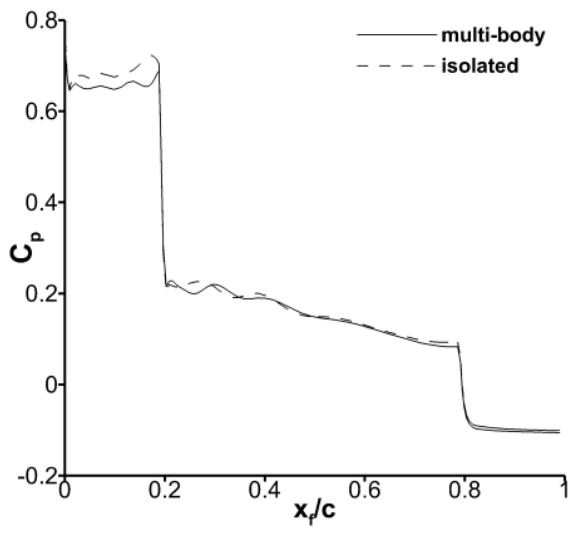

(b) chordwise pressure distribution on fin lower surface

\section{Fig. 28 Calculated receiver fin surface pressure distributions at $y_{f} / b=0.5$ : sharp generator, $\Delta z_{w} / D=2.94, \sigma_{R}=8^{\circ}$ $\sigma_{\mathrm{G}}=0^{\circ}$. Note the different $\mathrm{y}$-axis scales.}

\section{Conclusions}

The aerodynamics of a finned slender body in close proximity to a second generator body have been investigated through a series of wind tunnel tests and supporting computational fluid dynamic simulations. This builds on previous research considering an un-finned body. Overall, the interference effects are found to be highly complex as well as bespoke to each configuration and consequently general trends are difficult to extract.

However, it is possible to say that large interference loads are generally found when the diffracted shock is close to the fins. Although the fins provide a vital role in ensuring aerodynamic stability, in some cases they can have a detrimental influence on the interference characteristics of the receiver body. For example, in some configurations, the interference loads caused by large forces on the fins will induce a large pitch-up moment towards the generator body, thus increasing the likelihood of a collision between the two bodies. Furthermore and equally as importantly, the interference effects in some configurations are strong enough to cause the receiver to become statically unstable. This is an important finding since the body could potentially become uncontrollable.

The magnitude and sign of the interference loads are most sensitive to the axial impingement location of the primary disturbance. In some cases a change in sign is observed over the range of axial stagger configurations considered. Moreover, when the diffracted shock passes close to the leading edge of the lifting fins, there is an induced flow pitch which then results in large fin interference loads. 
The fin interference loads are a complex function of the three interference mechanisms discussed in this paper: the post-shock flow angularity and the two pressure-based mechanisms due to the impinging shock and expansion waves. The dominant interference mechanism which influences the fin loads is found to change over the axial stagger and receiver incidence range considered. This gives an indication of the complexity of the problem. The receiver incidence setting subtly plays a more significant role than in an un-finned configuration. This is because it is important which part of the receiver body is placed in which portion of the disturbance flowfield. For example, large loads can develop on the fins when the body is at high negative incidence and the fins are in close proximity to the generator body and therefore in the strongest region of the disturbance flowfield.

Overall, the measured and calculated results showed good agreement throughout, including detailed surface pressure validation between CFD and PSP. Moreover, the CFD calculations were successfully used to interpret the measured results and better understand the underlying flow physics of the problem. The CFD was particularly crucial in understanding the contribution of the fins to the overall interference loads since the wind tunnel model was unable to measure the fin forces and moments due to size constraints.

\section{Acknowledgments}

The authors would like to thank the UK MOD Defence Science and Technology Laboratory for both funding and providing technical support for this research. The first author was also partially funded through an Industrial CASE award from the UK Engineering and Physical Sciences Research Council.

\section{References}

${ }^{1}$ Wilcox, F., "Separation Characteristics of Generic Stores from Lee Side of an Inclined Flat Plate at Mach 6", NASA TM$4652,1995$.

${ }^{2}$ Hung, C., "Computation of Three-Dimensional Shock Wave and Boundary-Layer Interactions”, NASA TM-86780, 1985.

${ }^{3}$ Gapcynski, J., Carlson, H., “A Pressure-Distribution Investigation of the Aerodynamic Characteristics of a Body of Revolution in the Vicinity of a Reflection Plane at Mach Numbers of 1.41 and 2.01”, NACA RM-L54J29, 1955.

${ }^{4}$ Cenko, A., Waskiewicz, J., "Recent Improvements in Prediction Techniques for Supersonic Weapon Separation”, AIAA Journal of Aircraft, Vol. 20, No. 8, 1983, pp. 659-666.

${ }^{5}$ Newman, G., Fulcher, K., Ray, R., Pinney, M., “On the Aerodynamics/Dynamics of Store Separation from a Hypersonic Aircraft”, AIAA Paper 1992-2722, 1992.

${ }^{6}$ Chaplin, R., “Aerodynamic Interference between High-Speed Slender Bodies”, Ph.D. Thesis, Cranfield University, UK, 2009.

${ }^{7}$ Perkins, Jr., S.C. and Dillenius, M.F.E., “Supersonic Submunition Aerodynamics During Dispense," AIAA Journal of Spacecraft and Rockets, Vol. 28, No. 3, 1991, pp. 276-283. 
${ }^{8}$ Deep, R.A., Brazzel, C.E., and Sims, J.L., “Aerodynamics of Submunitions during Dispense,” AIAA Paper 1985-0105, 1985.

${ }^{9}$ Volkov, V., Derunov, E., "Interaction of a Combination of Bodies in Supersonic Flow Interference and Diffraction of Shock Waves in Flow Over Two Bodies of Revolution", Journal of Engineering Physics and Thermophysics, Vol. 79, No. 4, 2006, pp.712-721.

${ }^{10}$ Malmuth, N., Shaleav, V., "Theoretical Modelling of Interaction of Multiple Slender Bodies in Supersonic Flows", AIAA Paper 2004-1127, 2004.

${ }^{11}$ Gapcynski, J., "The Aerodynamic Characteristics of a Body in the Flow Field Near the Tip of a Circular-Arc Wing of Rectangular Plan Form at a Mach Number of 2.01”, NASA TM X-211, 1960.

${ }^{12}$ Waskiewicz, J., DeJongh, J., Cenko, A., “Application of Panel Methods to External Stores at Supersonic Speeds”, AIAA Journal of Aircraft, Vol. 20, No. 2, 1983, pp. 153-158.

${ }^{13}$ Fedorov, A., Malmuth, N., Soudakov, V., "Supersonic Scattering of a Wing-Induced Incident Shock by a Slender Body of Revolution”, Journal of Fluid Mechanics, Vol. 585, 2007, pp. 305-322.

${ }^{14}$ Derunov, E., Zheltovodov, A., Maksimov, A., "Development of Three-Dimensional Turbulent Separation in the Neighborhood of Incident Crossing Shock Waves", Thermophysics and Aeromechanics, Vol. 15, No. 1, 2008, pp. 29-54.

${ }^{15}$ Fedorov, A., Soudakov, V., Malmuth, N., "Theoretical Modeling of Two-Body Interaction in Supersonic Flow", AIAA Journal, Vol. 48, No. 2, 2010, pp. 258-266.

${ }^{16}$ Brosh, A., Kussoy, M., “An Experimental Investigation of the Impingement of a Planar Shock Wave on an Axisymmetric Body at Mach 3", NASA TM 84410, 1983.

${ }^{17}$ Morkovin, M., Migotsky, E., Bailey, H., Phinney, R., "Experiments on Interaction of Shock Waves and Cylindrical Bodies at Supersonic Speeds", Journal of Aerospace Science, Vol. 19, No. 4, 1952, pp. 237-247.

${ }^{18}$ Hung, C., "Impingement of an Oblique Shock Wave on a Cylinder", AIAA Paper 1982-0025, 1982.

${ }^{19}$ Brosh, A., Kussoy, M., Hung, C., "Experimental and Numerical Investigation of a Shock Wave Impingement on a Cylinder", AIAA Journal, Vol. 23, No. 6, 1985, pp. 840-846.

${ }^{20}$ Cenko, A.,"Store Separation Lessons Learned During the Last 30 Years", 27th International Congress of the Aeronautical Sciences, Nice France, 19-24 $4^{\text {th }}$ September 2010.

${ }^{21}$ Keen, S. et al., "Trajectory Simulations Should Match Flight Tests and Other Lessons Learned in 30 Years of Store Separation Analysis”, AIAA Paper 2009-0099. 2009.

${ }^{22}$ Chaplin, R., MacManus, D., Birch, T., “Aerodynamic interference between high-speed slender bodies”, Shock Waves, Vol. 20, No. 2, 2010, pp. 89-101.

${ }^{23}$ Chaplin, R., MacManus, D., Leopold, F., Martinez, B., Gauthier, T., Birch, T., "Experimental Investigation into the Interference Aerodynamics of Two Slender Bodies in Close Proximity”, Experiments in Fluids, Vol. 50, No. 2, 2011, pp. 491507.

${ }^{24}$ Chaplin, R., MacManus, D., Leopold, F., Martinez, B., Gauthier, T., Birch, T., "Computational and Experimental Investigation into Aerodynamic Interference Between Slender Bodies in Supersonic Flow”, Computers and Fluids, Vol. 5o, No. 1, 2011, pp. 155-174.

${ }^{25}$ Taylor, J., An Introduction to Error Analysis, University Science Books, California USA, 1997.

${ }^{26}$ Cobalt LLC, Cobalt Users Manual, 2007.

${ }^{27}$ Strang, W., Tomaro, R., Grismer, M., “The Defining Methods of Cobalt 60 : A Parallel, Implicit, Unstructured Euler/NavierStokes Solver”, AIAA Paper 1999-0786, 1999.

${ }^{28}$ Menter, F., “Zonal Two Equation k-w Turbulence Models for Aerodynamic Flows”, AIAA Paper 1993-2906, 1993.

${ }^{29}$ Pointwise, Gridgen, 2006. 
${ }^{30}$ Cummings, R., Morton, S., McDaniel, D., "Experiences in Accurately Predicting Time-Dependent Flows", Progress in Aerospace Sciences, Vol. 44, No. 4, 2008, pp. 241-257.

${ }^{31}$ Roache, P., Verification and Validation in Computational Science and Engineering, Hermosa Publishers, New Mexico USA, 1998.

${ }^{32}$ Moore, F., "Engineering codes for aeroprediction: state-of-the-art and new methods", Special Course of Missile Aerodynamics, AGARD-R-804, AGARD, Neuilly Sur Seine, France, 1994.

${ }^{33}$ Chin, S., Missile configuration design, $1{ }^{\text {st }}$ Edition, McGraw-Hill, New York, 1961, p279. 
2016-04-28

\section{Aerodynamic Interference on Finned Slender Body}

Chaplin, Ross

American Institute of Aeronautics and Astronautics

Ross Chaplin, David MacManus, Friedrich Leopold, Bastien Martinez, Thibaut Gauthier, and Trevor Birch. Aerodynamic Interference on Finned Slender Body, AIAA Journal, Vol. 54, No. 7 (2016), pp. 2017-2033.

http://dx.doi.org/10.2514/1.J054704

Downloaded from Cranfield Library Services E-Repository 